TI 2012-032/1

Tinbergen Institute Discussion Paper

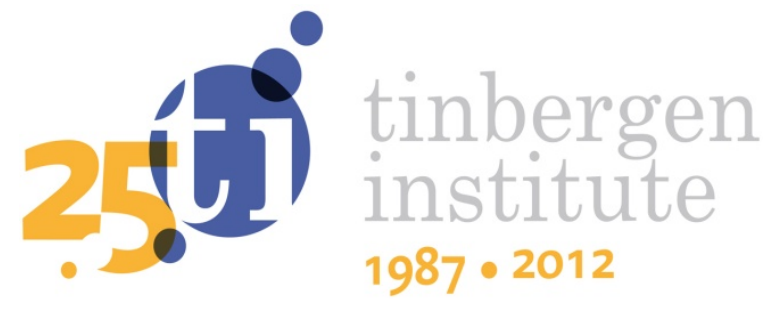

Networks and Collective Action

\author{
Ramon Flores ${ }^{1}$ \\ Maurice Koster ${ }^{2}$ \\ Ines Lindner 3 \\ Elisenda Molina'
}

\footnotetext{
' Universidad Carlos III de Madrid;

2 Faculty of Economics and Business, University of Amsterdam;

3 Faculty of Economics and Business Administration, VU University Amsterdam.
} 
Tinbergen Institute is the graduate school and research institute in economics of Erasmus University Rotterdam, the University of Amsterdam and VU University Amsterdam.

More TI discussion papers can be downloaded at http://www.tinbergen.nl

Tinbergen Institute has two locations:

Tinbergen Institute Amsterdam

Gustav Mahlerplein 117

1082 MS Amsterdam

The Netherlands

Tel.: +31(0)205251600

Tinbergen Institute Rotterdam

Burg. Oudlaan 50

3062 PA Rotterdam

The Netherlands

Tel.: +31(0)10 4088900

Fax: $+31(0) 104089031$

Duisenberg school of finance is a collaboration of the Dutch financial sector and universities, with the ambition to support innovative research and offer top quality academic education in core areas of finance.

DSF research papers can be downloaded at: http://www.dsf.nl/

Duisenberg school of finance

Gustav Mahlerplein 117

1082 MS Amsterdam

The Netherlands

Tel.: +31(0)20 5258579 


\title{
Networks and Collective Action
}

\begin{abstract}
This paper proposes a new measure for a group's ability to lead society to adopt their standard of behavior, which in particular takes account of the time the group takes to convince the whole society to adopt their position. This notion of a group's power to initiate action is computed as the reciprocal of the resistance against it, which is in turn given by the expected absorption time of a related finite state partial Markov chain that captures the social dynamics. The measure is applicable and meaningful in a variety of models where interaction between agents is formalized through (weighted) binary relations. Using Percolation Theory, it is shown that the group power is monotonic as a function of groups of agents. We also explain the differences between our measure and those discussed in the literature on Graph Theory, and illustrate all these concerns by a thorough analysis of two particular cases: the Wolfe Primate Data and the 11S hijackers' network.
\end{abstract}

Keywords: Collective action, Social networks, Influence and diffusion models, Network intervention, Group centrality measures

\section{Introduction}

Collective action sometimes seems to appear over night, e.g., large protests against a particular regime suddenly bring onto the streets thousands and thousands of people as happened in the Arabic Spring in 2011. Given a social network, which individuals or groups of individuals are able to ignite a chain-reaction and cause collective action on a large scale? Most importantly, how quickly does the collective behavior arise? What kinds of ties of a network are most important for collective action and what features are especially relevant? Somewhat analogously, which individuals in a network should we address if the aim is to prevent action of many when the action is harmful (as e.g. terrorist activity)? This kind of questions arises in many different areas, such as sociology and social psychology (e.g. [37], [27], [34]), epidemiology (e.g. [20], [56]), economics (e.g. [3], [12], [36], [44], [49], [53], [58]), social choice (e.g. [16],[17]), computer sciences (e.g. [23], [41], [47]) or systems reliability (e.g. [2]), covering the analysis of riot behavior, innovation and rumor diffusion, propaganda, strikes, consumption, network externalities, spread of fashions, migration, runs on banks, voting power, cascading failures in power systems, etc.

We select a model to describe how the action profile of a society evolves over time taking into account several elements that the systemic phenomena of collective behavior have in common. As posed by the mathematical sociologist Coleman (1990): (i) they involve a large number of people carrying out the same or similar actions at the same time; (ii) the system 
is dynamic and might eventually reach an equilibrium state; (iii) there is some kind of synchronization of actions, so individuals do not act independently; and (iv) there is some degree of unpredictability, sometimes leading to "explosive" results. Then, based on this kind of dynamical model, we propose two new indices of group power which carry the spirit of Coleman's (1971) power measures, i.e., the power to initiate action and the power to prevent action, respectively. Here, the power to initiate action will be our main focus as the other measure naturally follows from its definition. The power to initiate action quantifies a group's ability to trigger the society to adopt its behavior, and, if at all, how quickly individuals learn this mode of behavior. With this information, a social planner, a political party, or a firm's management may be able to actively address the most powerful individuals and thus help to prevent or to stimulate action in a given context. ${ }^{1}$

It is worth noting that our proposal of group power to initiate action may also be interpreted as a group centrality measure in the sense of Everett and Borgatti (1999), but its scope is wider and not restricted to a graph-theoretic context. In addition to allowing asymmetric binary relations between agents, it also relates the strength of a tie to the time it takes to reach consensus at the level of society. We will discuss the similarities and differences between graph-theoretical notions and ours for cases in which comparison makes sense - see Sections 4 and 6.

In order to define a dynamical model of collective action it is crucial to understand the role of the social structure in the sharing of information and the formation of opinions. We will assume that the society members are connected through a social network which is the primary conduit of information, opinions, and behaviors. More precisely, we adopt the interactive Markov chains (non-symmetric) model, in which an individual's choice of actions may depend on arbitrary neighborhoods of others. At each date, agents communicate with their neighbors in the social network and copy one of their actions from the last period according to a (updated) probabilistic rule. This approach agrees with the voter model, also called the invasion process, and first introduced along with their dual process called coalescing random walks in Holley and Liggett (1975), and also independently in Clifford and Sudbury (1973). Moreover, originally developed in the context of interacting stochastic processes, the voter model has been considered as a model of social interaction, as for instance by Asavathiratham (2000) and Even-Dar and Shapira (2007). Ni, Xie and Liu (2010) also consider an interactive Markov (non-symmetric) chain based on a monotonic ${ }^{2}$ modification of the voter model, which they called the incremental chance model.

Other social dynamics models which are related to our point or view are the following:

- Threshold models of diffusion of innovations, where individuals are assumed to have dif-

\footnotetext{
${ }^{1}$ See, e.g., Rogers (1995) on the important role of opinion leaders in the dissemination of information and their influence on opinions and decisions in various environments like marketing, social programs, education, campaigning, and others.

${ }^{2}$ In which it is assumed that once an agent has adopted an innovation he sticks with it.
} 
ferent thresholds that determine whether they will adopt an innovation as a function of the number (or proportion) of others in the population who have already adopted $\mathrm{it}^{3}$. Kempe, Kleinberg and Tardos (2005) generalize these models by introducing decreasing cascade models, in which a behavior spreads in a cascading fashion according to a probabilistic rule, beginning with a set of initially active agents, which are the individuals that have adopted the innovation.

- Interactive Markov chains (symmetric) models, in which the next state of an individual depends on his current state and on the current frequency distribution of the population among the states modeling the different positions. The dynamics of these models where first considered by Conlisk (1976), where it was introduced the concept of an interactive Markov chain as a framework for stochastic flows when the effects on the decisions of individuals of imitation, fashion, popularity, contagion, and so on cannot be ignored. The work of Stadje (1997) is a deep generalization of the Conlisk model. However, although the symmetric case provides insight into broad patterns of social behavior, it does not incorporate the micro-details of who interacts with whom. To incorporate networked interactions, a richer non-symmetric structure is needed.

- Opinion formation models, also called social influence network models by Friedkin (2001), which are models of information transmission, opinion formation, and consensus formation. Social influence network theory (Friedkin, 1991, 1998, 1999; Friedkin and Johnsen, 1990, 1997, 1999) includes, as special cases, French's formal theory of social power (French, 1956; Harary, 1959) and the seminal DeGroot's consensus formation model (DeGroot, 1974; Chatterjee and Seneta, 1977; Berger, 1981). The basic building block of these models is the fact that network influences are captured by a network effects model, in which each individual's attitude is influenced by his exposure, measured as a weighted average of the attitudes of the other members. Network effects model goes back to Erbing and Young (1979), with important contributions by Doreian (1982), and specially by Friedkin and Johnsen (see the previously referenced papers), and more recently by Valente (2005). In some sense, the dynamical process we adopt is also a network effects model, since it is based on the notion of exposure. However, whereas all these models describe the dynamics of opinions and are in fact deterministic, the voter model we have adopted describes the dynamics of actions ${ }^{4}$ and it is probabilistic in nature. The implications of this difference over the power group measures we propose is analyzed in detail in Section 4.

The remainder of the paper is organized as follows. Section 2 is devoted to a general pre-

\footnotetext{
${ }^{3}$ The dynamics of these models were first studied by Schelling (1971) and Granovetter (1978). For more recent work, see Valente $(1995,1996)$, Watts (2002), Dodds and Watts (2005), Lopez-Pintado and Watts (2008), and Young (2009).

${ }^{4}$ That is, we calculate a person's adoption status at each period of time, which is referred to (see Valente, 2010) as event history analysis (Allison, 1984).
} 
sentation of the dynamic model we have adopted to describe how the propensity to act in a society evolves over time; in the description of the model, we will follow the approach of Asavathiratham (2000). In Section 3, based on this model, we propose two indices of group power (power of a group to initiate action and power to prevent action). Section 4 is devoted to analyze the relation of the group power to initiate action with other centrality measures, while in Section 5 we prove that group power to initiate full action satisfies a compelling monotonicity property, i.e., if a set of agents is expanded its power to initiate full action will (weakly) increase. Finally, in Section 6 we apply the proposed measures to analyze some illustrative examples in detail, namely the behavior of a population of primates and the structure of the hijackers network in the 11S. In particular, we compare our power measure to standard centrality scores.

\section{The model: influence dynamics as a finite Markov chain}

Assume that society consists of finitely many members, represented by the set $N=\{1,2, \ldots, n\}$, which are connected through a social network whose flow of available information is captured, in some sense, by a weight matrix $\mathbf{S}$. For instance, in a binary symmetric context, $\mathbf{S}$ can be the adjacency matrix of the non-directed graph which models the connection between the agents. In this case, this matrix is usually referred as the sociomatrix. Nevertheless, in a different design it can model other features of the network like relational, positional and central properties through which agents are interlinked. It may as well incorporate exogeneous information which does not depend on the structure of the network. The reader is referred to Valente (2005, 2010) where a number of different ways of designing the weight matrix are proposed.

Based on the information contained in S, we derive the Influence Matrix, i.e., a stochastic ${ }^{5}$ matrix $\mathbf{W}$ whose elements are understood as influence parameters, $w_{i j}$ being the weight that agent $i$ assigns to agent $j$. Influence can be asymmetric, as for example if they are one-sided with $w_{i j}>0$ while $w_{j i}=0$. There are many ways to derive the matrix $\mathbf{W}$ from $\mathbf{S}$, and usually the former is defined in terms of some normalization process applied to the latter. As Friedkin (2010) points out: "different measures of the accorded weights have been employed in the operationalization (measurement models) of social influence network theory. The theory is open with respect to the definition of these weights". We discuss different constructions of the influence matrix in Section 6.

From now on, assume that $\mathbf{S}$ and $\mathbf{W}$ are fixed. The influence matrix $\mathbf{W}$ defines a (weighted) directed graph $D(\mathbf{W})=(N, E, W)$ with agents as nodes and $\operatorname{arcs}(i, j) \in N \times N:(i, j) \in E \Leftrightarrow$ $w_{i j}>0$, and $w_{i j}$ being the weight of arc $(i, j)$. If $w_{i j}>0$ then $j$ directly influences $i$, and if there is

\footnotetext{
${ }^{5} \mathrm{~A} n \times n$ matrix $\mathbf{Q}=\left(q_{i j}\right)_{i}^{j}$ is stochastic if $q_{i j} \geq 0$ for all $i, j=1,2, \ldots, n$, and $\sum_{j=1}^{n} q_{i j}=1$ for all $i=1,2, \ldots, n$.
} 
a directed path $P[i, j]$ from $i$ to $j$ in $D(\mathbf{W})^{6}$, then $j$ is under indirect influence of $i$. Note that $D(\mathbf{W})$ is not necessarily the directed weighted graph which defines the weight matrix $\mathbf{S}$, although the underlying structure of the graph (not the weights) is usually the same.

\subsection{Markov Chain}

In our context, the diffusion of information behaves like a stochastic process in discrete time, which we model through the network effects model approach and, more concretely, the network exposure models defined by Burt (1987) and Marsden-Friedkin (1993) ${ }^{7}$. The analysis of diffusion phenomena in social influence through network exposure, and particularly influence matrices, has attracted much attention because of its extreme flexibility. In the words of Valente, "Virtually any theoretical mechanism of social influence can be modeled". This study applies to a wide range of collective behavior phenomena where agents have a binary choice: to take action (being active) or not to take action (remain passive), characteristics indicated by 1 and 0 , respectively. From a dynamic point of view, we assume that the status of the agent may change as time goes by (see the event history analysis in Allison (1984)). At each date, agents communicate with their neighbors in the social network and update their propensity to act. The updating process is simple: an agent's new propensity is determined by the weighted average of his/her neighbors' actions from the previous period. Then, at each date, the status of each agent $i \in N$ is determined by a probabilistic rule based on his personal network exposure, which defines the probability of agent $i$ taking action, given the previous decisions of his neighbors. Formally, consider the following definitions, where $\mathbf{W}$ is the influence matrix.

States of a society: A state of the society $(N, \mathbf{W})$ is a tuple $x^{N} \in \sigma(N):=\{0,1\}^{n}, x_{i}^{N} \in\{0,1\}$ being the state of agent $i$, and $i=1, \ldots, n$, where $n$ is the cardinality of $N$. Special states are $\mathbf{z}^{N}, \mathbf{e}^{N} \in \sigma(N)$, according to which respectively none or everyone in $N$ take action. We will unambiguously identify $x^{N} \in \sigma(N)$ with a vector in $\mathbb{R}^{n}$. For $x^{N} \in \sigma(N)$, let $A\left(x^{N}\right):=\{i \in N \mid$ $\left.x_{i}^{N}=1\right\}$ the set of active agents in $N$.

Influence dynamics: For each society $(N, \mathbf{W})$, let $p: \sigma(N) \rightarrow[0,1]^{n}$ be the mapping defined by

$$
p\left(x^{N}\right)=\mathbf{W} \cdot x^{N}, \quad \text { for all } x^{N} \in \sigma(N) .
$$

Then for each state $x^{N}$ of society $(N, \mathbf{W})$ the number $p_{i}\left(x^{N}\right)$ represents the probability that $i$ takes action upon observing state $x^{N}$ of $(N, \mathbf{W})$.

\footnotetext{
${ }^{6}$ A directed path $P[i, j]$ from $i$ to $j$ in $D(\mathbf{W})$ is a subgraph of $(N, E)$ consisting of a sequence of nodes $\left\{i_{1}, \ldots, i_{r}\right\}$, and $\operatorname{arcs}\left(i_{1}, i_{2}\right),\left(i_{2}, i_{3}\right), \ldots,\left(i_{r-1}, i_{r}\right)$, where $i_{1}=i$ and $i_{r}=j$. Alternatively, we shall sometimes refer to a directed path as a set of (sequence of) arcs (of nodes) without any explicit mention of the nodes (without explicit mention of arcs).
}

${ }^{7}$ See Valente (2010) for a thorough treatment of this topic. 
Transition probabilities: Now, for $x^{N}, y^{N} \in \sigma(N)$ we define $m_{x^{N} y^{N}}$ as the conditional probability of state $x^{N}$ turning into state $y^{N}$, given by

$$
m_{x^{N} y^{N}}:=P\left\{X_{t+1}=y^{N} \mid X_{t}=x^{N}\right\}=\prod_{i \in A\left(y^{N}\right)} p_{i}\left(x^{N}\right) \prod_{j \in N \backslash A\left(y^{N}\right)}\left(1-p_{j}\left(x^{N}\right)\right),
$$

where $X_{t}^{N}=\left(X_{1 t}^{N}, \ldots, X_{n t}^{N}\right)$ is the random vector which describes the state of the society $(N, \mathbf{W})$ at time $t \in\{0,1,2, \ldots\}$.

Markov chain: Then, $\mathcal{M}=\left\{X_{t}^{N}\right\}_{t \geq 0}$ is a discrete time Markov chain with transition matrix $\mathbf{M}$ given by $\left(M_{i j}\right)_{i j}=\left(m_{s_{i}^{N} s_{j}^{N}}\right)_{i j}$, for all $i, j=1,2, \ldots, 2^{n}$, where the states are ordered according to the lexicographical order. Then $\sigma(N)=\left\{s_{1}^{N}, s_{2}^{N}, \ldots, s_{2^{n}}^{N}\right\}$, and $s_{1}^{N}=\mathbf{z}^{N}, s_{2^{n}}^{N}=\mathbf{e}^{N}$.

Unlike classical network measures we will not derive our group power measure from $\mathbf{S}$ nor from $\mathbf{W}$, but from the transition matrix $\mathbf{M}$. This is in sharp contrast to the usual social influence network models, in which the proposed scores are usually obtained by direct computations with the weight matrix and/or the influence matrix.

\subsection{Preliminaries: Partial Markov chain}

The main goal is to measure the impact on the society when the members of a subsociety $T \subseteq N$ confine themselves to act. The remaining agents start in a passive state. How long does it take until the active state of $T$ spreads over to the whole society (collective action) - and will this always happen eventually? In terms of our dynamic model the question is whether the associated Markov chain is absorbing and, if it is, to determine the expected absorption time. In order to address these questions in the following section we need to tailor the preceding definitions to subsocieties.

The actions of non-members of $T$ are governed by the above defined updating process. Let $s^{T} \in \sigma(T):=\{0,1\}^{t}$, where $t=|T|$ is the cardinality of $T$, be the state of subsociety $T \subseteq N$ that describes the position of agents in $T$ during the whole process, then the social dynamics of the subsociety $N \backslash T \subseteq N$ are described by the following partial Markov chain $\mathcal{M}\left(s^{T}\right)=\left\{X_{t}^{N \backslash T}\left(s^{T}\right)\right\}_{t \geq 0}$. Let us first adapt the previous definitions to this scenario.

Notation:. Each combination of $s^{T} \in \sigma(T)$ and $x^{N \backslash T} \in \sigma(N \backslash T)$ defines a state $\left[s^{T}, x^{N \backslash T}\right] \in \sigma(N)$ by

$$
\left[s^{T}, x^{N \backslash T}\right]_{i}= \begin{cases}s_{i}^{T}, & \text { if } i \in T, \\ x_{i}^{N \backslash T}, & \text { if } i \in N \backslash T .\end{cases}
$$

Influence dynamics on subsocieties: For each subsociety $T \subset N$, and for any given state $s^{T} \in \sigma(T)$, let $p^{s^{T}}: \sigma(N \backslash T) \rightarrow[0,1]^{n-t}$ be the mapping defined by

$$
p^{s^{T}}\left(x^{N \backslash T}\right)=\mathbf{W} \cdot\left[s^{T}, x^{N \backslash T}\right] \text {, for all } x^{N \backslash T} \in \sigma(N \backslash T)
$$


Transition probabilities: As before, for each state $x^{N \backslash T}$ of subsociety $N \backslash T$ the number $p_{i}^{s^{T}}\left(x^{N \backslash T}\right)$ represents the probability that $i \in N \backslash T$ takes action upon observing state $\left[s^{T}, x^{N \backslash T}\right]$ of $N$. Now, we define $m_{x^{N \backslash T} y^{N \backslash T}}\left(s^{T}\right)$ as the conditional probability of state $x^{N \backslash T}$ turning into state $y^{N \backslash T}$, given by

$$
\begin{aligned}
m_{x^{N \backslash T} y^{N \backslash T}}\left(s^{T}\right) & :=P\left\{X_{t+1}=\left[s^{T}, y^{N \backslash T}\right] \mid X_{t}=\left[s^{T}, x^{N \backslash T}\right] \text { and } X_{t+1}^{T}=s^{T}\right\}= \\
& =\prod_{i \in A\left(y^{N \backslash T}\right)} p_{i}^{s^{T}}\left(x^{N \backslash T}\right) \prod_{j \in N \backslash\left(T \cup A\left(y^{N \backslash T}\right)\right)}\left(1-p_{j}^{s^{T}}\left(x^{N \backslash T}\right)\right) .
\end{aligned}
$$

Partial Markov chain: Then, $\mathcal{M}\left(s^{T}\right)=\left\{X_{t}^{N \backslash T}\left(s^{T}\right)\right\}_{t \geq 0}$ is a discrete time Markov chain with transition matrix $\mathbf{M}\left(s^{T}\right)=\left(m_{s_{i}^{N \backslash T} s_{j}^{N \backslash T}}\left(s^{T}\right)\right)_{i j}$, for all $i, j=1,2, \ldots, 2^{n-t}$, where the states are labeled according to the lexicographical order. Thus, $\sigma(N \backslash T)=\left\{s_{1}^{N \backslash T}, s_{2}^{N \backslash T}, \ldots, s_{2^{n-t}}^{N \backslash T}\right\}$, with $s_{1}^{N \backslash T}=\mathbf{z}^{N \backslash T}, s_{2^{n-t}}^{N \backslash T}=\mathbf{e}^{N \backslash T}$.

\section{Power to initiate full action: definition and examples}

The properties of the (partial) Markov chain will depend on the particular state $s^{T}$, and the particular influence matrix $\mathbf{W}$ structure. In order to propose a method to measure $T$ 's power to initiate (and to prevent) action we focus on two special but natural cases: when agents in $T$ confine themselves to take action $\left(s^{T}=\mathbf{e}^{T}\right)$, and the opposite one, when agents in $N \backslash T$ confine themselves to never take action $\left(s^{T}=\mathbf{z}^{T}\right)$.

We will refer to the active subsociety $T \subseteq N$ as the hard core active group in the sense that they are incapable of change. The chain $\mathcal{M}\left(\mathbf{e}^{\mathcal{T}}\right)$ starts with members outside the hard core group in a passive state, $s_{1}^{N \backslash T}=\mathbf{z}^{N \backslash T}$. The resistance of the society to $T$ is then defined as the expected total time spent in some of the transient states $\left\{s_{1}^{N \backslash T}, s_{2}^{N \backslash T}, \ldots, s_{2^{n-t-1}}^{N \backslash T}\right\}$ before reaching the absorbing state $s_{2^{n-t}}^{N \backslash T}=\mathbf{e}^{N \backslash T}$, if at all.

Definition 1. Given $(N, \mathbf{W})$ and $T \varsubsetneqq N$, the resistance of the society to group $T$ action is the number $r(T) \in \mathbb{N} \cup\{\infty\}$ defined by

$$
r(T):=\mathbb{E}\left\{t \mid X_{t}^{N \backslash T}\left(\mathbf{e}^{T}\right)=\mathbf{e}^{N \backslash T} \text { and } X_{t-1}^{N \backslash T}\left(\mathbf{e}^{T}\right) \neq \mathbf{e}^{N \backslash T}\right\} .
$$

For $T=N$, the resistance of the society to group $N$ action is defined to be $r(N)=0$.

In particular, the higher the expected time that a group needs to move society into the all active absorbing state, the higher the resistance against this group. ${ }^{8}$ Note that $r(T)=\infty$ applies

\footnotetext{
${ }^{8}$ This definition is extendable to other levels of collective action, say, when a majority of action is the level in question. Here, the transition matrix $\mathcal{M}\left(\mathbf{e}^{\mathcal{T}}\right)$ can be adjusted by defining states as absorbing in which more than $50 \%$ of the society is active.
} 
when there is a group of agents $C \subseteq N \backslash T$ that is closed with respect to $\mathbf{W}$, i.e., $i \in C$ and $w_{i j}>0$ implies $j \in C$. Then in this situation (in $\mathcal{M})\left(\mathbf{e}^{T}\right)$ the state $\mathbf{e}^{N \backslash T}$ is not reachable from $\mathbf{z}^{N \backslash T}$. If there are no closed groups, $\mathcal{M}\left(\mathbf{e}^{T}\right)$ has two classes, namely $\mathcal{T}=\left\{s_{1}^{N \backslash T}, s_{2}^{N \backslash T}, \ldots, s_{2^{n-t-1}}^{N \backslash T}\right\}$ and $\mathcal{R}=\left\{\mathbf{e}^{N \backslash T}\right\}$, the first class being transient and the second recurrent. Here, the Markov chain $\mathcal{M}\left(\mathbf{e}^{T}\right)$ is recurrent with an absorbing state, and we can use standard Markov Chain Theory results to calculate $1 \leq r(T)<\infty$, in particular by means of the fundamental matrix $\mathbf{D}\left(\mathbf{e}^{T}\right)$ for $\mathcal{M}\left(\mathbf{e}^{T}\right)$. This matrix is given by $\mathbf{D}\left(\mathbf{e}^{T}\right)=\left(\mathbf{I}_{2^{n-t-1}}-\mathbf{Q}\right)^{-1}$, where $\mathbf{Q}\left(\mathbf{e}^{T}\right)$ is obtained from the transition matrix $\mathbf{M}\left(\mathbf{e}^{T}\right)$ deleting its last row and its last column. The first row of the corresponding fundamental matrix $\mathbf{D}\left(\mathbf{e}^{T}\right)$ gives the expected number of times that the process is in a transient state if it is started in the transient state $s_{1}$. In our setting this is the state in which only the hard core is active. The resistance of the society to group $T$ is therefore computed by the sum of the first row of the fundamental matrix:

$$
r(T)=\sum_{j=1}^{2^{n-t-1}} d_{1 j}\left(\mathbf{e}^{T}\right) .
$$

Of course, we are aware of the difficulties in tracking the full joint distribution of the Markov chain $\mathcal{M}\left(\mathbf{e}^{T}\right)$, which is defined by means of a $2^{n-t} \times 2^{n-t}$ transition matrix. However, the resistance to a group can be estimated by means of simulating the behavior of the corresponding Markov chain. This is precisely the method we propose to deal with our proposal in Section 6.

Finally, we interpret the power of the group $T$ to initiate full action as the inverse of the resistance against it.

Definition 2. The power of $T$ to initiate full action is defined as the reciprocal of $r(T)$,

$$
P(T)= \begin{cases}0, & \text { if } r(T)=\infty, \\ 1 / r(T), & \text { if } r(T)<\infty .\end{cases}
$$

In analogy to Definition 2, the power to prevent action of group $T \subseteq N$ refers to how quickly individuals learn to become inactive when the group acts as a hard core passive group (they remain forever inactive). Therefore, this power is given by the expected time it takes that none is in an active state, given that all agents in $T$ are passive all the time and the complementary group starts with a pure action profile. According to our model, then, the positive influence of every agent to push the remaining agents to take action equals the negative influence of every agent to push the remaining agents to drop action, and both questions have the same answer: the potential of actors to ignite a chain-reaction coincides with their potential to act as a firewall.

Remark 1. The absence of closed groups in $N \backslash T$ is equivalent to the property of $\mathbf{W}$ that each agent is directly or indirectly influenced by some agent in T. Another way to put this is in terms of the corresponding (weighted) directed graph $D(\mathbf{W})=(N, E, W)$. Let us denote by $\mathcal{I}(T) \subseteq N \backslash T$ the set of agents that are directly or indirectly influenced by some agent in $T$, that is,

$$
\mathcal{I}(T)=\{i \in N \backslash T \quad \mid \exists j \in T \text { with } P[i, j] \text { in } D(\mathbf{W})\}
$$


Then, there exists a closed set $C \subseteq N \backslash T$ if, and only if, $\mathcal{I}(T) \varsubsetneqq N \backslash T$. Therefore, in particular if the social structure of the society is described by a strongly connected network (i.e., for all $i, j \in N$ there exists a directed path $P[i, j]$ from $i$ to $j$ in $D(\mathbf{W})), r(T)$ is finite for all $T$ and $P(T)>0$.

We would like to stress that, apart from the hard core group, we do not consider the dynamics as a one-way process where agents do not leave an active state. This is in crucial distinction to the traditional innovation literature where - once adopted - individual agents stick with new innovations. We agree with Young (2003) when he asserts that: "Yet the same feedback mechanisms that cause innovations to be adopted also cause them to be abandoned ... Thus, if we want to know how long it takes, in expectation, for a "new" behavior to replace an old one, we must analyze the balance of forces pushing the adoption process forward (to become active agents, in our setting) on the one hand, and those pushing it back (to remain passive agents) on the other".

Note that in the case of symmetric relations, unless there are separate connected components, group $T$ action must eventually dominate for every group $\varnothing \neq T \subseteq N$. However, it could take "too much time" in practice. ${ }^{9}$ Moreover, eventual domination of the hard core group is no longer guaranteed when we consider asymmetric relations. This is illustrated in the following example.

Example 1. let $(N, \mathbf{W})$ be the society defined by $N=\{1,2,3,4,5\}$ and influence matrix

$$
\mathbf{W}=\left(\begin{array}{ccccc}
1 & 0 & 0 & 0 & 0 \\
0.9 & 0.1 & 0 & 0 & 0 \\
0 & 0.9 & 0 & 0.1 & 0 \\
0 & 0 & 0.1 & 0.8 & 0.1 \\
0.9 & 0 & 0 & 0.1 & 0
\end{array}\right)
$$

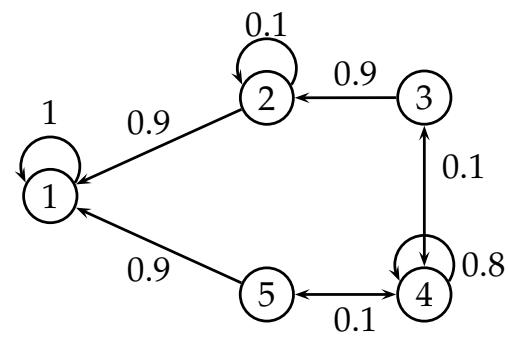

Since $C=\{1\}$ is closed relative to $\mathbf{W}$, then $r(T)=\infty$ (and therefore its power to initiate full action is 0 ) for all $T \subseteq N \backslash\{1\}$. Otherwise, whenever $1 \in T$, this effectively influences every agent in $N$, so that $r(T)$ is finite and the power of $T$ to initiate full action is positive. One may calculate $r(\{1\})=7.4252, r(\{1,2\})=6.8601, r(\{1,2,3\})=5.8905, r(\{1,2,3,4\})=1$, $r(\{1,2,4\})=1, r(\{1,2,3,5\})=5, \ldots$

Now assume the social network changes to the following:

\footnotetext{
${ }^{9}$ As Even-Dar and Shapira (2007) point out, to convince society quickly is crucial to the early stages of introducing a new technology into the market.
} 


$$
\mathbf{W}=\left(\begin{array}{ccccc}
0.8 & 0 & 0 & 0 & 0.2 \\
0.9 & 0.1 & 0 & 0 & 0 \\
0 & 0.9 & 0 & 0.1 & 0 \\
0 & 0 & 0.1 & 0.8 & 0.1 \\
0.1 & 0.9 & 0 & 0 & 0
\end{array}\right)
$$

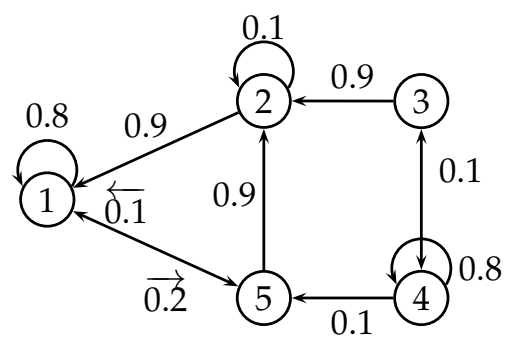

Here, $C=\{1,2,5\}$ is a unique closed group relative to $\mathbf{W}$ and, therefore, $r(T)=+\infty$ for all $T \subseteq\{3,4\}$.

By nature, our idea of society resistance to full action is addressed to understand the dynamics of connected networks ${ }^{10}$. However, it may happen that we deal with fragmented societies, made up of independent subsocieties $\left\{\left(N_{1}, \mathbf{W}_{1}\right), \ldots,\left(N_{m}, \mathbf{W}_{m}\right)\right\}$ that evolve independently. In this case, it is mandatory that group $T$ has some member of each subsociety in order to be able to disseminate action across the whole society. At this point, society resistance to group $T$ can be also defined by (1), or we can adopt an alternative definition and calculate resistance as the maximum of the resistance of the independent subsocieties. The first option may be adopted if we are interested in measuring partial action ${ }^{8}$. However, if we are concerned with full action and we want that the agents in each subsociety do not impose any externality over agents in the remaining ones, then resistance must be calculated as the maximum resistance, i.e.,

$$
r(T)=\max \left\{r\left(T_{1}\right), \ldots, r\left(T_{m}\right)\right\},
$$

where $r\left(T_{k}\right)$ is the society resistance to group $T_{k}=T \cap N_{k}$ in the corresponding subsociety $\left(N_{k}, \mathbf{W}_{k}\right)$, for all $k=1, \ldots, m$.

Example 2. Let $(N, \mathbf{W})$ be the society defined by $N=\{1,2,3,4,5\}$ and influence matrix

$$
\mathbf{W}=\left(\begin{array}{ccccc}
0 & 1 & 0 & 0 & 0 \\
0 & 0 & 1 & 0 & 0 \\
\frac{1}{2} & 0 & \frac{1}{2} & 0 & 0 \\
0 & 0 & 0 & \frac{1}{2} & \frac{1}{2} \\
0 & 0 & 0 & \frac{1}{2} & \frac{1}{2}
\end{array}\right)
$$

Then, $r(T)=\infty$ (and therefore its power to initiate full action is 0 ) for all $T \subseteq\{1,2,3\}$ or $T \subseteq\{4,5\}$. Otherwise, $T$ can influence every agent in $N$, and therefore $r(T)$ can be calculated. For instance: $r(\{1,4\})=\frac{10}{3}, r(\{1,4,5\})=3$ and $r(\{1,3,5\})=2$, when applying definition (1). However, in this example the society is actually divided into two independent subsocieties $\left(N_{1}, \mathbf{W}_{1}\right)$ and $\left(N_{2}, \mathbf{W}_{2}\right)$, where $N_{1}=\{1,2,3\}, N_{2}=\{4,5\}$, and

$$
\mathbf{W}_{1}=\left(\begin{array}{ccc}
0 & 1 & 0 \\
0 & 0 & 1 \\
\frac{1}{2} & 0 & \frac{1}{2}
\end{array}\right), \quad \mathbf{W}_{2}=\left(\begin{array}{cc}
\frac{1}{2} & \frac{1}{2} \\
\frac{1}{2} & \frac{1}{2}
\end{array}\right),
$$

\footnotetext{
${ }^{10}$ We will say that two nodes $i$ and $j$ are connected in $D(\mathbf{W})$ if the graph contains at least one path (not necessarily directed) from node $i$ to node $j$.
} 
that evolve independently. Thus, if we do not want that agents in $T \cap N_{1}$ impose any externality over agents in $T \cap N_{2}$, and vice versa, then we must calculate $r(\{1,4\})=\max \left\{r_{1}(\{1\}), r_{2}(\{4\})\right\}=$ $\max \{3,2\}=3<\frac{10}{3}, r(\{1,4,5\})=\max \{3,0\}=3$ and $r(\{1,3,5\})=\max \{1,2\}=2$.

\section{Relation with centrality measures}

Recall that our group power measure can also be interpreted as a group centrality measure (Everett and Borgatti, 1999). The aim of this section is to compare our measure to other centrality measures. However, note that in contrast to resistance (a) not all standard network centrality measures can be used in signed and valued graphs, (b) not all of them can be extended to groups without invoking the reduced graph approach (Everett and Borgatti, 1999) ${ }^{11}$ and, finally, (c) not all of them are appropriate for influence type processes (Borgatti, 2005). Despite these difficulties, Borgatti (2005) shows that a conceptual comparison can be made by interpreting centrality measures as characteristics of flows through a network. Using this approach we conclude that our proposal as individual measure is not comparable, i.e. not proportional, to Freeman's closeness or betweenness. Rather, it is more consistent with eigenvalue-like measures. The canonical representation by Borgatti (2005) shows a characteristic of these measures: they assume trajectories of flows that can not only be circuitous, but also revisit nodes and lines multiple times along the way. In order to establish their relationship, the following result due to Stadje (1997) is crucial, since it allows us to interpret eigenvector-based centrality measures (Katz, 1953; Bonacich, 1972, 1987; and Brin and Page, 1998), and also the total effects centrality defined in Friedkin (1991), as probabilities of reaching consensus in a stochastic dynamic model based on the influence matrix $\mathbf{W}$.

If we allow for a set $T \subseteq N$ of initially active nodes to be deactivated during the process, then we will always work with the general Markov chain $\mathcal{M}=\left\{X_{t}^{N}\right\}_{t \geq 0}$ which is a recurrent chain with two absorbing states, namely $\mathbf{z}^{N}$ and $\mathbf{e}^{N}$, when the influence matrix $\mathbf{W}$ is irreducible. In that case (see Stadje, 1997), the probability of absorption in $\mathbf{e}^{N}$, given the initial state is $\left[\mathbf{e}^{T}, \mathbf{z}^{N \backslash T}\right]$, equals

$$
\pi_{\mathbf{e}^{N}}\left[\mathbf{e}^{T}, \mathbf{z}^{N \backslash T}\right]=\sum_{i \in T} \xi_{i}
$$

where $\left(\xi_{i}\right)_{i \in N}$ is the stationary distribution of the irreducible influence matrix $\mathbf{W}$. If $\mathbf{W}$ is not irreducible, then the society $(N, \mathbf{W})$ is fragmented into independent subsocieties and the probability of absorption can be calculated as the product of the corresponding absorption probabilities on each of the subsocieties. Therefore, from an individual point of view, eigenvaluelike measures are closely related to the individual power to initiate action. The eigenvalue of agent $k$ gives the probability of the new behavior replacing the old one when agent $k$ starts the

\footnotetext{
${ }^{11}$ Which replaces all members of a group by a single "super" vertex whose neighborhood is the union of the neighborhoods of all group members.
} 
innovative behavior, and taking account that even himself could abandon the new behavior; whereas his power to initiate action counts the time it takes to convince everybody else to adopt the innovation when he will never abandon it. ${ }^{12}$ However, the eigenvalue approach does not properly generalize to groups since it will result in an additive measure, which oversimplifies the complex structure of the underlying non-linear network dynamics.

Example 3. Consider a society of four members, where relations are symmetric and binary, and are described by the following graph, with adjacency matrix $\mathbf{A}$ :

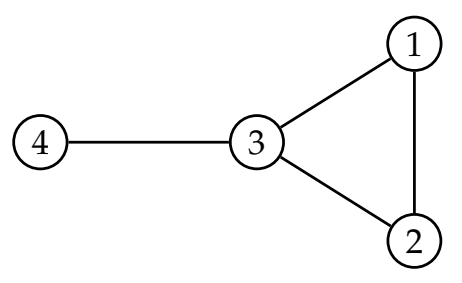

$$
\mathbf{A}=\left(\begin{array}{llll}
0 & 1 & 1 & 0 \\
1 & 0 & 1 & 0 \\
1 & 1 & 0 & 1 \\
0 & 0 & 1 & 0
\end{array}\right)
$$

In order to derive an influence matrix we modify its diagonal to include each agent's personal attitude towards his own opinions. The diagonal elements are indicative for an agent's independence. Suppose that each agent regards his own opinion equally important as that of all his direct relations (diagonal elements of 1 ). After normalization we arrive at the following influence matrix:

$$
\mathbf{W}=\left(\begin{array}{cccc}
1 / 3 & 1 / 3 & 1 / 3 & 0 \\
1 / 3 & 1 / 3 & 1 / 3 & 0 \\
1 / 4 & 1 / 4 & 1 / 4 & 1 / 4 \\
0 & 0 & 1 / 2 & 1 / 2
\end{array}\right)
$$

Intuitively, agent 3 should be the most powerful agent, whereas agent 4 should be the least powerful one. Agents 1 and 2, who occupy a symmetric position in the society, should have an intermediate strength.

Now, in order to calculate the power of agent 1, the state space of the partial Markov chain $\mathcal{M}\left(\mathbf{e}_{1}\right), \sigma(\{2,3,4\})$, will be given by the matrix

$$
\sigma(\{2,3,4\})=\left(\begin{array}{llllllll}
0 & 0 & 0 & 0 & 1 & 1 & 1 & 1 \\
0 & 0 & 1 & 1 & 0 & 0 & 1 & 1 \\
0 & 1 & 0 & 1 & 0 & 1 & 0 & 1
\end{array}\right)
$$

where each column represents a state vector of $\{2,3,4\}$. In the first state no agent in $N \backslash\{1\}$ is active and the vector $\mathbf{W e}_{1}$ indicates each agent's probability to act in the following period (apart from 1 who always stays active), in particular $p_{2}=1 / 3, p_{3}=1 / 4$ and $p_{4}=0$. The probability for the subsociety $\{2,3,4\}$ to stay in the first state where only 1 is active is hence

\footnotetext{
${ }^{12}$ Which fits precisely the terrorist's movements behavior. There is a hard-core of terrorist that are always pushing their revolutionary behavior. It is also convenient in those cases in which, for instance, a group of people are paid for wearing a specific clothing-brand.
} 
given by $\left(M\left(\mathbf{e}_{1}\right)\right)_{11}=\left(1-p_{2}\right)\left(1-p_{3}\right)\left(1-p_{4}\right)=1 / 2$. The remaining elements of $M\left(\mathbf{e}_{1}\right)$ are computed analogously. We get

$$
M\left(\mathbf{e}_{1}\right)=\left(\begin{array}{cccccccc}
\frac{1}{2} & 0 & \frac{1}{6} & 0 & \frac{1}{4} & 0 & \frac{1}{12} & 0 \\
\frac{1}{6} & \frac{1}{6} & \frac{1}{6} & \frac{1}{6} & \frac{1}{12} & \frac{1}{12} & \frac{1}{12} & \frac{1}{12} \\
\frac{1}{12} & \frac{1}{12} & \frac{1}{12} & \frac{1}{12} & \frac{1}{6} & \frac{1}{6} & \frac{1}{6} & \frac{1}{6} \\
0 & \frac{1}{12} & 0 & \frac{1}{4} & 0 & \frac{1}{6} & 0 & \frac{1}{2} \\
\frac{1}{6} & 0 & \frac{1}{6} & 0 & \frac{1}{3} & 0 & \frac{1}{3} & 0 \\
\frac{1}{24} & \frac{1}{24} & \frac{1}{8} & \frac{1}{8} & \frac{1}{12} & \frac{1}{12} & \frac{1}{4} & \frac{1}{4} \\
0 & 0 & 0 & 0 & \frac{1}{8} & \frac{1}{8} & \frac{3}{8} & \frac{3}{8} \\
0 & 0 & 0 & 0 & 0 & 0 & 0 & 1
\end{array}\right)
$$

The fundamental matrix of the absorbing system $D\left(\mathbf{e}_{1}\right)=\left(\mathbf{I}_{7}-Q\left(\mathbf{e}_{1}\right)\right)^{-1}$ turns to be:

$$
D\left(\mathbf{e}_{1}\right) \approx\left(\begin{array}{lllllll}
2.779 & 0.131 & 0.889 & 0.201 & 1.651 & 0.439 & 1.681 \\
0.907 & 1.333 & 0.645 & 0.448 & 0.947 & 0.479 & 1.167 \\
0.674 & 0.204 & 1.492 & 0.296 & 0.944 & 0.510 & 1.223 \\
0.203 & 0.180 & 0.165 & 1.452 & 0.256 & 0.362 & 0.376 \\
1.010 & 0.109 & 0.708 & 0.173 & 2.463 & 0.418 & 1.819 \\
0.459 & 0.143 & 0.422 & 0.310 & 0.677 & 1.388 & 1.109 \\
0.294 & 0.050 & 0.226 & 0.096 & 0.628 & 0.361 & 2.185
\end{array}\right)
$$

The expected time it takes until the action of agent 1 has triggered all others is the resistance of the society to 1 and given by

$$
\sum_{j=1}^{7} d\left(\mathbf{e}_{1}\right)_{1 j} \approx 2.779+0.131+0.889+0.201+1.651+0.439+1.681=7.771 .
$$

Analogously, all group's resistances and powers can be obtained. The following table compares resistance and power to different group centrality measures. The group eigenvalue centrality is obtained as the corresponding eigenvalue in the group reduced graph. In that case, the internal structure of the group has no effect. The same occurs when group closeness based on the minimum method ${ }^{13}$ and group degree measures are obtained. The group measure we propose does not show this drawback. Note that group resistance is the only measure that differentiates between all groups of two agents, up to symmetries of agents 1 and 2, whereas the remaining measures only distinguish one of them, and moreover, all of them give the same score for all groups of three agents.

\footnotetext{
${ }^{13}$ In which the distance to a group in a graph is defined as the minimum distance to each of its members. We select this method, rather than average or maximum group closeness since these are not monotonic measures. Moreover, our measure is much more related to minimum closeness than to the other two (see the analysis of primate data example in Section 6).
} 


\begin{tabular}{|l|lccccccccc|}
\hline GROUP & $\{1\}$ & $\{3\}$ & $\{4\}$ & $\{1,2\}$ & $\{1,3\}$ & $\{1,4\}$ & $\{3,4\}$ & $\{1,2,3\}$ & $\{1,2,4\}$ & $\{1,3,4\}$ \\
\hline RESISTANCE & 7.77 & 4.17 & 15 & 5.33 & 2.3 & 3.12 & 3.86 & 2 & 1.33 & 1.5 \\
POWER & 0.128 & 0.240 & 0.067 & 0.188 & 0.435 & 0.321 & 0.259 & 0.500 & 0.752 & 0.667 \\
\hline DEGREE & 2 & 3 & 1 & 1 & 2 & 2 & 2 & 1 & 1 & 1 \\
EIGENVALUE & 0.523 & 0.612 & 0.282 & 0.5 & 0.707 & 0.707 & 0.707 & 0.707 & 0.707 & 0.707 \\
BETWEENNESS & 0 & 2 & 0 & 0 & 1 & 0 & 0 & - & - & - \\
MIN-CLOSENESS & 4 & 3 & 5 & 3 & 2 & 2 & 2 & 1 & 1 & 1 \\
DEGREE-NORM. & 1.5 & 1 & 3 & 2 & 1 & 1 & 1 & 1 & 1 & 1 \\
BET.-NORM. & 0 & 1.5 & 0 & 0 & 2 & 0 & 0 & - & - & - \\
M-CLOSE.-N . & 0.75 & 1 & 0.6 & 0.667 & 1 & 1 & 1 & 1 & 1 & 1 \\
\hline
\end{tabular}

Table 1: Example 3 group centrality measures.

Whereas centralities like degree and closeness ${ }^{14}$ are defined by the number of ties between the agents, their generalizations to signed and valued graphs (see, e.g., Newman (2001), Brandes (2008)) mainly focus on tie weights instead. Our approach combines the two views in a natural way as we will illustrate below. See Opshal et al. (2010), where generalizations combining both aspects are defined by means of a weighted bi-criteria approach.

Moreover, when applied to non-symmetric relations for these generalizations it is easy to run into agents with infinite closeness, simply because some are out of reach. This is not a problem in our context, but it is worthwhile to note that we are dealing with a group measure and every agent, apart from the isolated ones, is always contained in a group that can actually reach all of the agents in the complementary group.

Example 4. (Opsahl et al., 2010) Consider a society of five members, where relations are symmetric and valued as depicted by the following weighted undirected graph with adjacency matrix A:

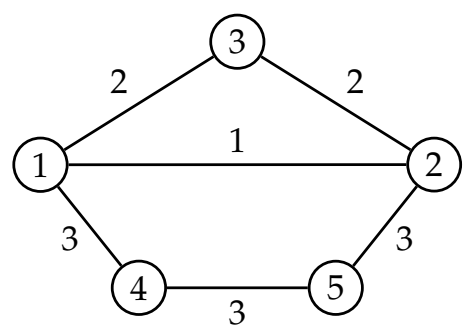

$$
\mathbf{A}=\left(\begin{array}{lllll}
0 & 1 & 2 & 3 & 0 \\
1 & 0 & 2 & 0 & 3 \\
2 & 2 & 0 & 0 & 0 \\
3 & 0 & 0 & 0 & 3 \\
0 & 3 & 0 & 3 & 0
\end{array}\right)
$$

Suppose an agent's self-influence outweighs the average influence by the direct relations, we

\footnotetext{
${ }^{14}$ We will not discuss the relation to betweenness measures because the properties of our measure are radically different.
} 
may take as $\mathbf{W}$ the normalization of $\mathbf{A}^{*}$ :

$$
\mathbf{A}^{*}=\left(\begin{array}{ccccc}
2 & 1 & 2 & 3 & 0 \\
1 & 2 & 2 & 0 & 3 \\
2 & 2 & 2 & 0 & 0 \\
3 & 0 & 0 & 3 & 3 \\
0 & 3 & 0 & 3 & 3
\end{array}\right) \quad \mathbf{W}=\left(\begin{array}{ccccc}
\frac{1}{4} & \frac{1}{8} & \frac{1}{4} & \frac{3}{8} & 0 \\
\frac{1}{8} & \frac{1}{4} & \frac{1}{4} & 0 & \frac{3}{8} \\
\frac{1}{3} & \frac{1}{3} & \frac{1}{3} & 0 & 0 \\
\frac{1}{3} & 0 & 0 & \frac{1}{3} & \frac{1}{3} \\
0 & \frac{1}{3} & 0 & \frac{1}{3} & \frac{1}{3}
\end{array}\right) .
$$

In this case, the power to initiate action of agents 1 and 2 is close to that of agents 4 and 5, but greater, reflecting the fact that agents 1 and 2 need less intermediary nodes in their geodesic paths to the remaining agents than 4 and 5 . However, the weighted closeness obtained by inverting the tie weights (Newman, 2001; Brandes, 2001) stresses the weight dependency by giving more centrality to agents 4 and 5 .

\begin{tabular}{|c|ccc|}
\hline AGENT & RESIST. & POWER & W-CLOSENESS \\
\hline 1 & 9.5829 & 0.1043 & 2.5 \\
3 & 14.4813 & 0.069 & 2.6667 \\
5 & 9.9126 & 0.1009 & 2.1667 \\
\hline
\end{tabular}

Table 2: Calculated power and weighted-closeness for the Opsahl (2010) example.

\section{Properties: monotonicity}

As non-monotone group power measures should be used with extreme caution, the question is whether the group measure we have defined is monotonic. We will state first some results of Percolation Theory, which are the main tool for proving the monotonicity of the resistance, as well as some notation. We refer the reader to Holley and Liggett (1975) for a thorough introduction to this topic.

\subsection{Percolation Theory}

Fix a society $(N, \mathbf{W})$. Given a subsociety $S \subseteq N$, let us define the weighted directed graph $D\left(\mathbf{W}_{S}\right)=\left(N, E, W_{S}\right)$, with agents as nodes and $\operatorname{arcs}(k, j) \in N \times N$, such that $w_{S}(k, j)>0$, where $w_{S}(k, j)=w_{k j}$, for all $k \notin S$ and $j \in N$, and $w_{S}(k, k)=1, w_{S}(k, j)=0$, for all $k \in$ $S$ and $j \neq k$. Then, the Markov process $\mathcal{M}\left(\mathbf{e}^{S}\right)$ defined by $S$ can be described in terms of an associated coalescing random walk (CRW) over $D\left(\mathbf{W}_{S}\right)$, which is another stochastic process defined as follows. At time 0 , every node holds a black dot. At every time step, the dot on node $i$ hops to one of its neighbors with the probability given on the arc. All dots are hopping 
simultaneously. When two or more dots land at the same node, they coalesce into one, and continue hopping.

We will adopt the alternative definition of the CRW process through the outcome matrix,

$$
U_{S}=\left\{\mathbf{y}^{*}=\left\{\left(y_{1}^{*}(t), \ldots, y_{n}^{*}(t)\right)\right\}_{t \geq 1} \mid y_{i}^{*}(t) \in N, \forall i \in N, \forall t \geq 1\right\},
$$

which describes the social dynamics by means of saying that at each time period, every agent chooses a neighbor at random with a probability determined by $W_{S}$, and then copies his/her status. Note that at each time period, agent $i$ decides who he/she wants to copy with the same set of probabilities regardless of his/her current status or its neighbors'. Here, $y_{i}^{*}(t) \in N$ is the neighbor that agent $i$ has decided to copy in period $t$. For a given outcome matrix $\mathbf{y}^{*}$, let $p_{i k}\left(\mathbf{y}^{*}\right)$ be the backward path originating from agent $i$ at time $k$ and terminating at some agent at time 0 . Let $\eta_{i k}\left(\mathbf{y}^{*}\right) \in N$ be that agent.

Let us assume now that $S \varsubsetneqq N, i_{0} \notin S$, and denote $S^{\prime}=S \cup\left\{i_{0}\right\}$, and by $T_{S}$ and $T_{S^{\prime}}$ the random times to reach the revolutionary state $\mathbf{e}^{N}$ when the initial states are $\mathbf{z}^{N \backslash S}$ and $\mathbf{z}^{N \backslash S^{\prime}}$ in $\mathcal{M}\left(\mathbf{e}^{S}\right)$ and $\mathcal{M}\left(\mathbf{e}^{S^{\prime}}\right)$, respectively. Moreover, let $\mathbf{y}^{N \backslash S^{\prime}}=\left\{y_{t}^{N \backslash S^{\prime}}\right\}_{t \geq 0}$ be a realization of $\mathcal{M}\left(\mathbf{e}^{S^{\prime}}\right)$ for which $T_{S^{\prime}}\left(\mathbf{y}^{N \backslash S^{\prime}}\right)=t_{0}$ for a certain time $t_{0}$, and let $\mathbf{y}^{*}$ be any outcome matrix that reproduces $\mathbf{y}=\left\{\left[y_{t}^{N \backslash S^{\prime}}, \mathbf{1}^{S^{\prime}}\right]\right\}_{t \geq 0}$. That is, $y_{i t}=0$ if $\eta_{i t}\left(\mathbf{y}^{*}\right) \notin S^{\prime}$, and $y_{i t}=1$ if $\eta_{i t}\left(\mathbf{y}^{*}\right) \in S^{\prime}$, for all agent $i \in N$, and for all $t \geq 1$. Then, the $n$ backward paths $p_{i t_{0}}\left(\mathbf{y}^{*}\right)$ of length $t_{0}$ that reproduce the adopting patterns from time $t=t_{0}$ to the initial time $t=0$ for every $i \in N$, end at some agent in $S^{\prime}$ (i.e., $\eta_{i t_{0}}\left(\mathbf{y}^{*}\right) \in S^{\prime}$, for all $i \in N$ ). Moreover, $t_{0}$ is the minimum time satisfying this condition.

Now, let us define the outcome matrix set $Y^{*}$ as the matrix composed by all the outcome matrices $\mathbf{y}^{*}$ that reproduce $\mathbf{y}$, and let

$$
Y^{*}\left(t_{0}\right)=\bigcup\left\{Y^{*} \mid \mathbf{y}^{N \backslash S^{\prime}} \text { with } T_{S^{\prime}}\left(\mathbf{y}^{N \backslash S^{\prime}}\right) \leq t_{0}\right\},
$$

for each $t_{0} \geq 1$. Then,

$$
P\left\{T_{S^{\prime}} \leq t_{0}\right\}=\sum_{\mathbf{y}^{*} \in Y^{*}\left(t_{0}\right)} P\left\{U_{S^{\prime}}=\mathbf{y}^{*}\right\}=\sum_{\mathbf{y}^{*} \in Y^{*}\left(t_{0}\right)} g_{S^{\prime}}\left(\mathbf{y}^{*}, t(\mathbf{y})\right),
$$

where

$$
g_{S^{\prime}}\left(\mathbf{y}^{*}, t(\mathbf{y})\right)=\prod_{i \notin S^{\prime}} \prod\left\{w_{S^{\prime}}(j, k) \mid(j, k) \in p_{i t(\mathbf{y})}\left(\mathbf{y}^{*}\right)\right\},
$$

and being $t(\mathbf{y})$ the time it takes the society to reach the revolutionary state when it is evolving according to $\mathbf{y}$ in $\mathcal{M}\left(\mathbf{e}^{S^{\prime}}\right)$. That is, $t(\mathbf{y})=T_{S^{\prime}}\left(\mathbf{y}^{N \backslash S^{\prime}}\right)$. Note that for every $i \in S^{\prime}$ (including $\left.i_{0}\right)$, and for every instant of time $t, y_{i}^{*}(t)=i$.

Now, for any given outcome matrix $\mathbf{y}^{*}$, we can define the set $X_{\mathbf{y}^{*}}^{*}$ whose elements are the matrices $\mathbf{x}^{*}$ whose elements are the outcome matrices for the partial Markov chain $\mathcal{M}\left(\mathbf{e}^{S}\right)$ such that $\mathbf{x}_{i}^{*}(t)=\mathbf{y}_{i}^{*}(t)$, for all $i \neq i_{0}$, for all $t \geq 1$. As abstract matrices, the elements of $X_{\mathbf{y}^{*}}^{*}$ differ from $\mathbf{y}^{*}$ only in the $i$-th row, whose entries can take different values in $N$. 
For every $\mathbf{x}^{*}$, we can of course define again:

$$
g_{S}\left(\mathbf{x}^{*}, t(\mathbf{x})\right)=\prod_{i \notin S} \prod\left\{w_{S}(j, k) \mid(j, k) \in p_{i t(\mathbf{x})}\left(\mathbf{x}^{*}\right)\right\},
$$

and the definition of $X_{\mathbf{y}^{*}}^{*}$ implies that

$$
g_{S^{\prime}}\left(\mathbf{y}^{*}, t(\mathbf{y})\right)=\sum_{\mathbf{x}^{*} \in X_{\mathbf{y}^{*}}^{*}} g_{S}\left(\mathbf{x}^{*}, t(\mathbf{x})\right) .
$$

Now we are ready to prove the desired result.

\subsection{Proof of the monotonicity}

Proposition 1. Let $(N, \mathbf{W})$ be a given society, and let $S$ and $T$ two subsocieties with $S \subseteq T$. Then $r(T) \leq r(S)$.

Proof. Let $\varnothing \neq S \varsubsetneqq N$ be a non-trivial subgroup of agents, and let $i_{0} \in N \backslash S$. Then, it is enough to compare the resistance of the society to $S$ to the one to $S \cup\left\{i_{0}\right\}$, and to check that the former should be, as intuition suggests, greater than the latter. Four cases are possible:

(i) If $S \cup\left\{i_{0}\right\}=N$, then $r(S) \geq r(N)=1$, which is the smallest resistance.

(ii) If $\mathcal{I}\left(S \cup\left\{i_{0}\right\}\right) \varsubsetneqq N \backslash\left(S \cup\left\{i_{0}\right\}\right) \Rightarrow \mathcal{I}(S) \varsubsetneqq N \backslash S$, and therefore $r\left(S \cup\left\{i_{0}\right\}\right)=r(S)=\infty$.

(iii) If $\mathcal{I}\left(S \cup\left\{i_{0}\right\}\right)=N \backslash\left(S \cup\left\{i_{0}\right\}\right)$, but $\mathcal{I}(S) \varsubsetneqq N \backslash S \Rightarrow r\left(S \cup\left\{i_{0}\right\}\right)<\infty=r(S)$.

(iv) Otherwise, $S \cup\left\{i_{0}\right\} \varsubsetneqq N, 1 \leq r(S)<\infty$, and $1 \leq r\left(S \cup\left\{i_{0}\right\}\right)<\infty$.

For the three first cases the result is clear, so let us analyze (iv) in detail. We will check that

$$
P\left\{T_{S} \leq t_{0}\right\} \leq P\left\{T_{S \cup i_{0}} \leq t_{0}\right\}, \forall t_{0} \geq 1,
$$

so that $E\left(T_{S}\right) \geq E\left(T_{S \cup i_{0}}\right)$.

The proof is based on the fact that the distribution of $T_{S}$ and $T_{S \cup i_{0}}$ in both Markov chains, $\mathcal{M}\left(\mathbf{e}^{S}\right)$ and $\mathcal{M}\left(\mathbf{e}^{S \cup i_{0}}\right)$, can be analyzed by means of their associated coalescing random walks, as seen in the previous section. If we adopt the notation introduced there, with $S^{\prime}=S \cup\left\{i_{0}\right\}$ in particular, we observe that $X_{\mathbf{y}^{*}}^{*}$ is disjoint from $X_{\mathbf{y}^{\prime *}}^{*}$ for every two different realizations $\mathbf{y}^{*}$ and $\mathbf{y}^{\prime *}$ of $\mathcal{M}\left(\mathbf{e}^{S^{\prime}}\right)$, so it follows from (3) and (4), that:

$$
P\left\{T_{S^{\prime}} \leq t_{0}\right\}=\sum_{\mathbf{y}^{*} \in Y^{*}\left(t_{0}\right)} g_{S^{\prime}}\left(\mathbf{y}^{*}, t(\mathbf{y})\right)=\sum_{\mathbf{y}^{*} \in Y^{*}\left(t_{0}\right)} \sum_{\mathbf{x}^{*} \in X_{\mathbf{y}^{*}}^{*}} g_{S}\left(\mathbf{x}^{*}, t(\mathbf{x})\right)
$$


The key point now is that every outcome matrix $\mathbf{x}^{*} \in \mathcal{M}\left(\mathbf{e}^{S}\right)$ such that $t(\mathbf{x}) \leq t_{0}$ belongs to exactly one set $X_{\mathbf{y}^{*}}^{*}$, the corresponding to the matrix $\mathbf{y}^{*} \in \mathcal{M}\left(\mathbf{e}^{S^{\prime}}\right)$ such that for every $t \leq 1$, $y_{i_{0}}^{*}(t)=i_{0}$, and $y_{i}^{*}(t)=x_{i}^{*}(t)$. According to the percolation structure, is is easy to realize that $t(\mathbf{y}) \leq t_{0}$, and the strict equality holds exactly when $t(\mathbf{y})$ is determined by the copy in $\mathcal{M}\left(\mathbf{e}^{S^{\prime}}\right)$ of a node in $S$.

Hence we have the inequality

$$
P\left\{T_{S} \leq t_{0}\right\}=\sum_{\mathbf{x}^{*} \in X^{*}\left(t_{0}\right)} g_{S}\left(\mathbf{x}^{*}, t(\mathbf{x})\right) \leq \sum_{\mathbf{y}^{*} \in Y^{*}\left(t_{0}\right)} \sum_{\mathbf{x}^{*} \in X_{\mathbf{y}^{*}}^{*}} g_{S}\left(\mathbf{x}^{*}, t(\mathbf{x})\right)=P\left\{T_{S^{\prime}} \leq t_{0}\right\} .
$$

Then $r\left(S^{\prime}\right)=E\left(T_{S^{\prime}}\right) \leq E\left(T_{S}\right)=r(S)$, and we are done with this case. Note that the inequality will be usually strict, as in general not all the elements $\mathbf{x}^{*}$ of $X_{\mathbf{y}^{*}}^{*}$, for any given $\mathbf{y}$, will be absorbed in a time smaller than $t_{0}$ in $\mathcal{M}\left(\mathbf{e}^{S}\right)$.

Note that if we adopt definition (2) to deal with fragmented societies, resistance remains monotonic. Let $(N, \mathbf{W})$ be a given society that is fragmented into $m$ independent subsocieties $\left\{\left(N_{1}, \mathbf{W}_{1}\right), \ldots,\left(N_{m}, \mathbf{W}_{m}\right)\right\}$. Let $S$ and $S \cup\left\{i_{0}\right\}$ be two groups in $N$ satisfying condition (iv), and let $k\left(i_{0}\right) \in\{1, \ldots, m\}$ be the subsociety which agent $i_{0}$ belongs to. Then $S_{k}=S \cap N_{k}=$ $\left(S \cup\left\{i_{0}\right\}\right) \cap N_{k}=\left(S \cup\left\{i_{0}\right\}\right)_{k}$, for all $k \neq k\left(i_{0}\right)$, and $\left(S \cup\left\{i_{0}\right\}\right)_{k\left(i_{0}\right)}=S_{k\left(i_{0}\right)} \cup\left\{i_{0}\right\}$. Then, taking into the previous general proof, it follows that

$$
\begin{aligned}
r(S) & =\max \left\{r\left(S_{1}\right), \ldots, r\left(S_{k\left(i_{0}\right)}\right), \ldots, r\left(S_{m}\right)\right\} \geq \\
& \geq \max \left\{r\left(S_{1}\right), \ldots, r\left(S_{k\left(i_{0}\right)} \cup\left\{i_{0}\right\}\right), \ldots, r\left(S_{m}\right)\right\}=r\left(S \cup\left\{i_{0}\right\}\right)
\end{aligned}
$$

\section{Comparative analysis}

In this section we present comparative statistics results concerning the choice of the weight matrix $\mathbf{S}$ and self-influence in particular. We do this by discussing two illustrative examples, that of Wolfe's Primate network and the terrorist network 11S. The focus will be on the relation of the group power measure with standard centrality scores (both individuals and for groups) and in which sense the former is more general than the latter; the modeling of the self-influence of an agent; the relationship with the network effects models and specially the wide range of possibilities allowed in the context of network exposure, and the interpretation as a network intervention, in the spirit of the key-player problem.

\subsection{Self-Influence}

The influence matrix $\mathbf{W}$ is the basic concept which not only defines relations between different agents, but also an agent's independence from his social environment; here the off-diagonal entries serve to summarize the relations between agents whereas the diagonal entries represent 
independence. As illustrated by Example 4, social network data often come encoded as binary (weighted) symmetric or asymmetric relations, leaving the diagonal entries blank. This implies degrees of freedom in interpreting the respective self-influences. Below, we will describe three ideas to add the information about self-influence when needed, and perform a sensitivity analysis of the resistance of the society to each group relative to these choices. As an example, we will describe the Wolfe's primate data discussed in Everett and Borgatti (1999), which deals with binary and weighted symmetric relations.

Let $\mathbf{S}$ be the weight matrix, which is the (weighted) adjacency matrix of the (weighted) undirected graph. We consider the following models for the diagonal:

- Symmetric and independent self-influences: assume the same fixed value for every agent's self influence ${ }^{15}$. We define:

$$
w_{i i}=\delta \in[0,1) \text {, and } w_{i j}=(1-\delta) \frac{s_{i j}}{\sum_{k=1}^{n} s_{i k}} \text {, for all } i, j \text {. }
$$

- Self-influences based on agent's direct friends: assume that each agent gives to his own opinion the same importance that he gives to his direct friends' opinion. In that case, we take:

$$
w_{i i}=\frac{\bar{s}_{i}}{\bar{s}_{i}+\sum_{k=1}^{n} s_{i k}} \text {, and } w_{i j}=\left(1-w_{i i}\right) \frac{s_{i j}}{\sum_{k=1}^{n} s_{i k}} \text {, for all } i, j,
$$

where $\bar{s}_{i}$ is the average influence that his direct friends exert over agent $i$, i.e., $\bar{s}_{i}=\frac{\sum_{j \neq i} s_{i j}}{\delta(i)}$, $\delta(i)$ being the degree of node $i$ in the corresponding undirected (weighted) graph defined by $\mathbf{S}$. See Example 3 for an application of this choice to a binary undirected graph, and Example 4 for its application to a weighted undirected graph.

- Self-influences based on agent's centrality in the social network: following Friedkin (2001), we will assume that the structural measure of an agent's susceptibility to influence, measured by $1-w_{i i}$, is based on the agent's centrality in the social network. Moreover, similar to that paper, we draw on the most elementary measure of centrality, the degree of the agent. Hence we write:

$$
w_{i i}=1-\sqrt{1-\frac{1}{1+e^{-(\delta(i)-2 \bar{\delta})}}}, \text { and } w_{i j}=\left(1-w_{i i}\right) \frac{s_{i j}}{\sum_{k=1}^{n} s_{i k}}, \text { for all } i, j,
$$

where $\bar{\delta}$ is the average degree of the agents, when relations are symmetric and binary.

\subsection{Wolfe Primate data: Self-Influence Sensitivity Analysis and Relation with group centrality measures}

In the sequel we undertake the analysis of the Wolfe primate data, which are given as a standard dataset in UCINET (Borgatti, Everett and Freeman, 2002). The data represent three months

\footnotetext{
${ }^{15}$ Since we do not have exogenous information to attach different values to different primates. We propose several asymmetric actions later.
} 
of interactions between a troop of monkeys, observed in the wild by Linda Wolfe as they sported by a river in Ocala, Florida. Joint presence at the river was coded as an interaction and these were summed within all pairs. The dataset also contains information about the sex and age of each animal. Everett and Borgatti (1999) consider six different groups to illustrate their group centrality proposals. Four of the groups were formed by age, and two formed by sex: (1) Age 14-16 (1,6,11,13,19); (2) Age 10-13 (2,3,8,12,16); (3) Age 7-9 (4,5,9,10,15,17); (4) Age $4-5(7,14,18,20)$; (5) Male (1 to 5); and (6) Female (6 to 20). They dichotomized the data, formerly symmetric and valued, by taking the presence of a tie if there were more than six interactions over the time period (see Figure 1). We will analyze the binary case to develop a comparative analysis, but also describe the valued case, to which our proposal can be properly applied. As in [24], when dealing with the binary case, we have permanently deleted the isolate nodes 2,6,16,18,19 and 20.

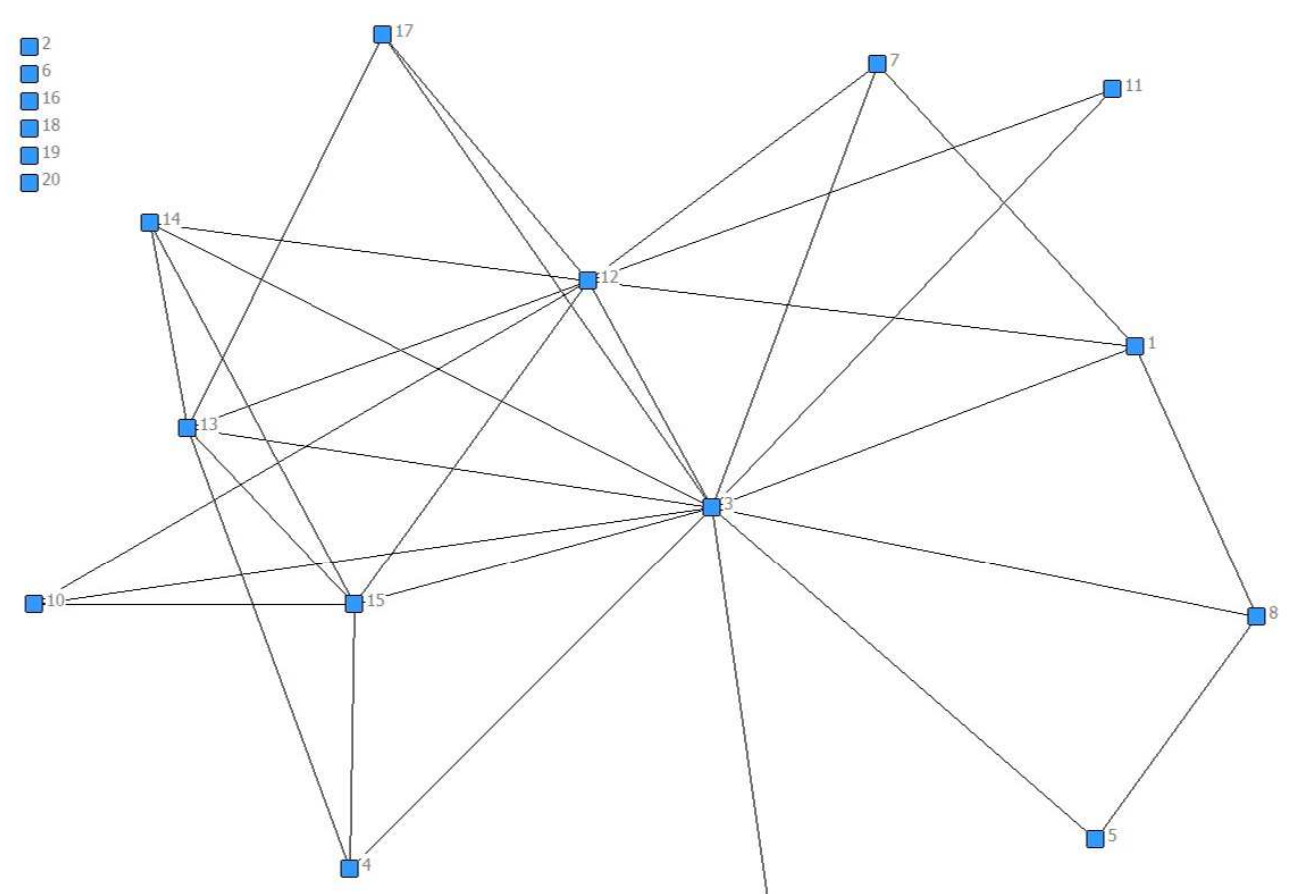

Figure 1: Wolfe Primate binary social network

Table 3 shows the group centrality scores for the six groups, as well as the resistance of the society to each one under the different scenarios we have considered: symmetric and independent self-influences (denoted by $R_{\text {delta }}$ ), with self-influence values from $\delta=0$ to $\delta=0.9$; self-influences based on agent's direct friends (denoted by $R_{\text {mean }}$ ), and self-influences based on agent's centrality in the social network $\left(R_{\text {degree }}\right)$. Note that it is possible to replace $\delta(i)$ by a parameter incorporating other aspects of autonomy as e.g. the relative time each primate does not encounter other primates or ethnologic knowledge to differentiate autonomy attitude according to, for example, age. However, this would require additional information. 


\begin{tabular}{|c|c|c|c|c|c|c|c|c|c|c|c|c|}
\hline \multirow[b]{2}{*}{ Group } & \multirow[b]{2}{*}{ Degree } & \multicolumn{3}{|c|}{ Closeness } & \multirow[b]{2}{*}{ Between. } & \multicolumn{5}{|c|}{ Fixed self-influences } & \multirow[b]{2}{*}{$R_{\text {mean }}$} & \multirow[b]{2}{*}{$R_{\text {degree }}$} \\
\hline & & Min. & Average & Max. & & $R_{0}$ & $R_{0.2}$ & $R_{0.4}$ & $R_{0.75}$ & $R_{0.9}$ & & \\
\hline Age 14-16 & 8 & 14 & 18 & 20 & 2.84 & 10.82 & 10.82 & 10.82 & 33.94 & 84.90 & 11.36 & 20.00 \\
\hline Age $10-13$ & 11 & 11 & 15 & 21 & 43.5 & 4.50 & 5.63 & 5.63 & 18.82 & 47.83 & 5.76 & 4.56 \\
\hline Age 7-9 & 5 & 11 & 13.7 & 15 & 0 & 7.27 & 8.85 & 8.85 & 27.63 & 68.99 & 8.85 & 16.04 \\
\hline Age $4-5$ & 5 & 19 & 20.5 & 22 & 0 & 15.58 & 18.57 & 18.57 & 56.07 & 139.14 & 19.25 & 31.33 \\
\hline Male & 10 & 10 & 16 & 20 & 24.34 & 6.11 & 7.51 & 7.51 & 23.93 & 60.11 & 7.52 & 6.62 \\
\hline Female & 4 & 4 & 6.4 & 7 & 0.5 & 2.16 & 2.88 & 2.88 & 10.30 & 26.36 & 2.96 & 9.39 \\
\hline
\end{tabular}

Table 3: Group centrality and the resistance of the society for the Primate data. Absolute values.

\begin{tabular}{|c|c|c|c|c|c|c|c|c|c|c|c|c|}
\hline \multirow[b]{2}{*}{ Group } & \multirow[b]{2}{*}{ Degree } & \multicolumn{3}{|c|}{ Closeness } & \multirow[b]{2}{*}{ Between. } & \multicolumn{5}{|c|}{ Fixed self-influences } & \multirow[b]{2}{*}{$R_{\text {mean }}$} & \multirow[b]{2}{*}{$R_{\text {degree }}$} \\
\hline & & Min. & Average & Max. & & $R_{0}$ & $R_{0.2}$ & $R_{0.4}$ & $R_{0.75}$ & $R_{0.9}$ & & \\
\hline Age 14-16 & 0.53 & 0.79 & 0.61 & 0.55 & 0.03 & 0.98 & 0.98 & 1.30 & 3.09 & 7.72 & 1.03 & 1.82 \\
\hline Age 10-13 & 0.73 & 1 & 0.73 & 0.52 & 0.41 & 0.41 & 0.51 & 0.69 & 1.71 & 4.35 & 0.52 & 0.41 \\
\hline Age 7-9 & 0.36 & 0.73 & 0.58 & 0.53 & 0 & 0.91 & 1.11 & 1.45 & 3.45 & 8.62 & 1.11 & 2.01 \\
\hline Age 4-5 & 0.31 & 0.63 & 0.59 & 0.55 & 0 & 1.30 & 1.55 & 2.00 & 4.67 & 11.60 & 1.60 & 2.61 \\
\hline Male & 0.67 & 1 & 0.63 & 0.5 & 0.23 & 0.61 & 0.75 & 0.99 & 2.39 & 6.01 & 0.75 & 0.66 \\
\hline Female & 0.8 & 1 & 0.63 & 0.57 & 0.05 & 0.54 & 0.72 & 1.00 & 2.57 & 6.59 & 0.74 & 2.35 \\
\hline
\end{tabular}

Table 4: Group centrality and the resistance of the society for the Primate data. Normalized values.

Group closeness depends on the definition of distance from the group to an outside node. Everett and Borgatti (1999) considered (i) the minimum distance to each of the group's nodes; (ii) the average of the distances; and (iii) the maximum distance.

Table 4 gives the corresponding normalized values (see [24]), which have greater significance in this context since we compare groups of different sizes. Here, resistance has been divided by the number of non-group members. This value can be interpreted as the average expected time to convince a non-group agent to become active.

All group measures but group closeness with the maximum method rank groups of Male, Female and Age 10-13 primates as the three best ones. Moreover, comparing group closeness (with the minimum and average method), degree and resistance results, we see a broad agreement between the measures. However, there are some interesting aspects in favor of resistance that should be remarked. With respect to the relation between closeness and resistance, first note that minimum and average closeness does not provide much sensitivity. All the three aforementioned groups attain the maximal value with the minimum method, whereas the average method does not differentiate among Male and Female groups. This is not the case with resistance. Given a group in active mode, which features of the network are relevant in order to achieve full action as soon as possible? Resistance indicates a change in the relative ordering among Male and Female groups with respect to different self-influence choices. When agents are very sensitive to influence, see $R_{0}, R_{0.2}$ and $R_{\text {mean }}$ (which is in between) results, the Female group is more powerful than the Male group. Note that the Male group contains primate 3 , who is related with all female primates. With decreasing independence he is more easily convinced, and reciprocally he becomes harder to convince with increasing independence $(\delta)$. 
Note also that the relative largest difference among these two groups is obtained for $R_{\text {degree }}$ (self-influences based on agent's centrality in the social network). In that case primate 3 becomes very hard to convince (with $w_{33}=0.875$ ), and therefore the resistance of the society to the Female group rises drastically. Moreover, note that the Female group is considerably larger in size (15) than the Males (5). This advantage, however, decreases as male primates become less sensitive to influence. To sum up, for small values of $\delta$ the Female group is more powerful than the Male group, which reproduces group degree and closeness relative order; whereas for bigger values of $\delta$ the Male group is more powerful reflecting the relative order of betweenness.

Obviously agents are harder to influence with increasing $\delta$, and therefore the resistance of the society to every group increases. Analyzing those increments, we observe that the marginal effects of increasing $\delta$ over resistance are bigger as $\delta$ approaches to one, and also are bigger for less powerful groups than they are for more powerful ones.

The analysis so far was based on dichotomous data (by taking the presence of a tie to be more than six interactions over the time period). We will now analyze the valued case. When the number of interactions over the three months time period is taken into account, we obtain a weighted undirected connected graph without isolated nodes. Table 5 shows the resistance of the society to each of the considered groups and indicates a change in the ranking of a group's power to initiate action. In contrast to the dichotomous model, the primates' group of ages ranging from 10 to 13 is not among the most powerful, and is ranked fourth in almost all scenarios. The Female group is now the most powerful group for all scenarios, except in the extreme cases in which all primates are very independent, with self-influence values of $\delta=0.75$ and $\delta=0.9$. Here, as before, the advantage of larger group size decreases as male primates become less sensitive to influence, and Female group's power falls from rank one to the third position for $\delta=0.75$, and even to the fourth for $\delta=0.9$.

Let us consider next self-influences based on agent's centrality in the social network. Replacing degree on (7) by weighted degree, the self-influences we obtain are zero for all primates but primate 3, whose high level of independence prevents this effect (with $w_{33}=0.9992$ ). As a result, society resistance's to all groups not containing primate 3 increases considerably, the resistance to the Female group above all. If we obtain the self-influences through the degree ${ }^{16}$, the Female group remains as the most powerful group, but the Male group loses some of its power favoring the Age 7-9 group.

\subsection{Selecting the Weight Matrix}

In the previous example we analyzed the correlation of resistance with some well-known group centrality measures. Moreover, according to different criteria, we proposed different choices of

\footnotetext{
${ }^{16}$ Then we must consider the difference $\delta(i)-\bar{\delta}$, instead of $\delta(i)-2 \bar{\delta}$, which turns all monkeys totally sensitive to influence $\left(w_{i i}=0\right.$, for all $\left.i\right)$.
} 


\begin{tabular}{|c|c|c|c|c|c|c|c|c|}
\hline & \multicolumn{5}{|c|}{ Fixed self-influences } & & & \multirow{2}{*}{ Group } \\
\cline { 2 - 6 } & $R_{0}$ & $R_{0.2}$ & $R_{0.4}$ & $R_{0.75}$ & $R_{0.9}$ & $R_{\text {mean }}$ & $R_{w-\text { degree }}$ & $R_{\text {degree }}$ \\
\hline Age 14-16 & 0.61 & 0.75 & 0.98 & 2.34 & 5.85 & 0.64 & 105.82 & 14.42 \\
Age 10-13 & 0.55 & 0.68 & 0.89 & 2.14 & 5.37 & 0.58 & 0.55 & 12.94 \\
Age 7-9 & 0.51 & 0.64 & 0.85 & 2.05 & 5.17 & 0.54 & 107.28 & 11.32 \\
Age 4-5 & 0.80 & 0.97 & 1.26 & 2.95 & 7.32 & 0.84 & 114.34 & 19.32 \\
Male & 0.51 & 0.63 & 0.84 & 2.03 & 5.09 & 0.54 & 0.51 & 12.32 \\
Female & 0.41 & 0.57 & 0.81 & 2.11 & 5.43 & 0.45 & 249.76 & 3.92 \\
\hline
\end{tabular}

Table 5: Normalized group centrality and the resistance of the society for the valued Primate data.

the diagonal of the weight matrix, and explored the consequences of the respective definitions. This subsection shows how to take a somewhat deeper profit of the freedom in the choice of this matrix by adding information which not depend directly, as in the classical (symmetric) case, on the the number of incident edges in each vertex. In the spirit of the network exposure models, and according to the recommendations of Valente (see comments on Section 2 about this point), we will consider different weight matrices to derive the influence matrix conducting the dynamical process. As a result, computing the resistance of the society to each group in each of the considered scenarios provides different group power measures which are not only based on individual direct relations, but also include information derived from the total network structure. Moreover, this approach allows to introduce exogeneous information which does not depend on the structure of the network.

The forthcoming subsection applies these ideas to the terrorist network of the 11S, which deals with binary symmetric relations. We will now describe the different influence matrices to be considered. Assume the underlying graph is connected, and consider first the following symmetric sociomatrices:

- The matrix $\mathbf{S}(1)$ is the adjacency matrix of the graph.

- The matrix $\mathbf{S}(2)$ is obtained by inverting the entries $d_{i j}$ of the distance matrix $D$, for $i \neq j$. Recall that the entry $d_{i j}$ of this matrix is the geodesic distance between the node $i$ and the node $j$. For every $i$, we define $S(2)_{i i}=0$.

- The matrix $\mathbf{S}(3)$ is obtained squaring the entries of $\mathbf{S}(2)$.

- The matrix $\mathbf{S}(4)$ emerges from $\mathbf{S}(3)$ by setting all entries with $a_{i j}<1 / 9$ equal to zero.

- If the elements of the matrix $\mathbf{G}=\{g\}_{i j}$ count the number of geodesical paths between each pair of nodes $i$ and $j$, we define $\mathbf{S}(5)$ as the matrix of pondered geodesical paths, whose entries are $\mathbf{S}(5)_{i j}=g_{i j} / d_{i j}$, if $i \neq j$, and zero otherwise.

The matrices $\mathbf{S}(2), \mathbf{S}(3), \mathbf{S}(4)$ and $\mathbf{S}(5)$ take the distance between nodes into account. In $\mathbf{S}(3)$ the influence of the distance is weakened in comparison to $\mathbf{S}(2)$. In $\mathbf{S}(4)$ we do not consider any relation between nodes which are separated by a distance bigger than three, while in $\mathbf{S}(5)$ 
the influence of distance is weighted by the number of geodesic paths connecting the nodes. It is easy to imagine similar versions of these matrices.

Finally, we consider two different kinds of non-symmetric matrices:

- Assume that there exists a directed valued graph with more relational information between the agents in the social network, and call $\mathbf{E}$ the weight matrix. Define $\mathbf{S}(6)$ such that $\mathbf{S}(6)_{i j}=\mathbf{S}(1)_{i j}+\mathbf{E}_{i j}$, for every $i \neq j$, and zero otherwise. In an analogous way, $\mathbf{S}(7)$ is defined as $\mathbf{S}(7)_{i j}=\mathbf{S}(6)_{i j}$ if $\mathbf{S}(1)_{i j} \neq 0$, and zero otherwise. Here, $\mathbf{S}(6)$ always takes account of the weights. For $\mathbf{S}(7)$ this is only the case when there is an edge joining the corresponding nodes in the original relational graph.

- Let $C$ be any individual centrality measure, and let $C_{i}$ be agent $i$ 's centrality. Then $\mathbf{S}(k)$, for $k=8,9,10$, are matrices obtained when the influence of agent $i$ over the remaining agents is weighted according to its centrality score. In that case, $\mathbf{S}(k)_{i j}=C_{j} \mathbf{S}(1)_{i j}$, for all $i, j$. For $k=8,9,10, C$ will be respectively the normalized degree, the normalized closeness or the normalized betweenness.

The above definitions represent different versions of the weight matrix, up to fixing the own individual level of independence (i.e., up to fixing the diagonal of the $\mathbf{S}(\cdot)$ matrices). In this analysis, we have assigned a certain proportional weight to the influence that an agent exerts to itself. As we explained in the previous section, this is not the only possible choice for the elements of the diagonal; however, it is quite reasonable from the point of view of the dynamical model we adopt, as it assumes that the number of times an agent adopts his own previous action is an average of the number of times he adopts the action of those agents influencing him. Now, we must derive the influence matrices in a reasonable way from the defined sociomatrices. As we want to preserve the information as far as possible, we derive the influence matrix $\mathbf{W}$ by means of a normalization process. In particular, if $\mathbf{S}$ is a matrix $n \times n$, then, according to (6), we define:

$$
w_{i j}=\frac{\widetilde{s}_{i j}}{\sum_{k=1}^{n} \widetilde{s}_{i k}}, \text { for all } i, j,
$$

where $\widetilde{s}_{i j}=s_{i j}$, for all $i \neq j, s_{i i}=\frac{\sum_{j \neq i} s_{i j}}{\delta_{+}(i)}$, and being $\delta_{+}(i)$ the out-degree of node $i$ in the corresponding (directed) weighted graph defined by $\mathbf{S}$.

We will describe the implications of the different choices of these matrices in the next example.

\subsection{The 11S network}

In the sequel we undertake an analysis of the terrorist network of the 11S. Our starting point is the version of the network in Figure 2, whose links come from terrorist that lived or learned 
together (black edges) as well as some temporary links that were only activated just before the attack in order to coordinate the cells. See Krebs (2002) for further information.

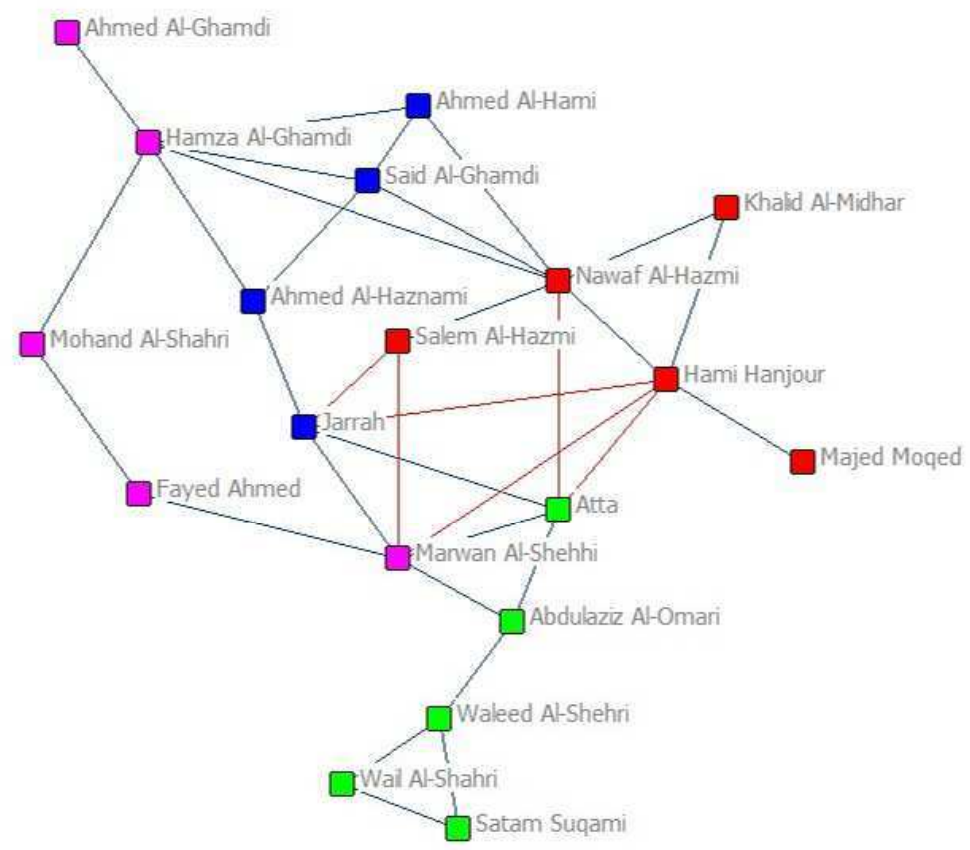

Figure 2: 11S social network

We constructed the weight matrices derived from that graph and the corresponding influence matrices according to the definitions above. ${ }^{17}$ Note that the mere structure of the network allows to construct all the matrices except for the cases $\mathbf{S}(6)$ and $\mathbf{S}(7)$ (see below), which require exogenous information in order to construct a valued graph. We have introduced extra information corresponding to other discovered contacts between the hijackers, as for example registration in the same hotels, renting the same car or share the same gym. As a result, the value on an edge increases with reported contacts between the hijackers defining it. For each choice, we have computed the resistance of the societys to each individual terrorist and described the most influential group. The numerical method is an estimation of a group's power to initiate full action by means of stochastic simulation, and we adopt a natural greedy algorithm to identify the 4 -members ${ }^{18}$ leader group. The idea is to begin with the terrorist with the smallest resistance, and proceed with an induction: given a group $S$ of terrorists, select the terrorist $\{i\}$ who produces the biggest reduction in the resistance of the society when added to

\footnotetext{
${ }^{17}$ The dataset is available on request.

${ }^{18}$ As it is well known, the nineteen hijackers which prepared and executed the attack were distributed in four cells, one for each plane that they planned to crash. Flight AA\#11, crashed into WTC North, in green colour; Flight AA\#77, crashed into Pentagon, in red; Flight UA\#11, crashed in Pennsylvania, in blue; and Flight UA\#173, crashed into WTC South, in pink.
} 


\begin{tabular}{|c|c|c|c|c|c|c|c|c|c|c|c|}
\hline Hard core & $\mathbf{S}(1)$ & $\mathbf{S}(2)$ & $\mathbf{S}(3)$ & $\mathbf{S}(4)$ & $\mathbf{S}(5)$ & $\mathbf{S}(6)$ & $\mathbf{S}(7)$ & $\mathbf{S}(8)$ & $\mathbf{S}(9)$ & $\mathbf{S}(10)$ & \\
\hline $1^{\text {st }}$ to enter & Al-Shehhi & N. Al-Hazmi & N. Al-Hazmi & N. Al-Hazmi & Al-Shehhi & Atta & Atta & N. Al-Hazmi & N. Al-Hazmi & Atta \\
\hline $2^{\text {nd }}$ to enter & N. Al-Hazmi & Al-Shehhi & Al-Shehhi & Al-Shehhi & Jarrah & N. Al-Hazmi & Al-Shehhi & Al-Shehri & Al-Omari & Al-Omari \\
\hline $3^{\text {rd }}$ to enter & Al-Shehri & H. Al-Ghamdi & H. Al-Ghamdi & Atta & Hanjour & Al-Shehri & H. Al-Ghamdi & Al-Shehhi & Al-Shehhi & N. Al-Hazmi \\
\hline $4^{\text {th }}$ to enter & H. Al-Ghamdi & Jarrah & Al-Shehri & H. Al-Ghamdi & Al-Haznawi & H. Al-Ghamdi & Hanjour & H. Al-Ghamdi & W. Al-Shahri & Al-Shehhi \\
\hline
\end{tabular}

Table 6: Determining the hard core

group $S$. We will refer to the sequentially selected terrorists as "the hard core". The results of the greedy algorithm are depicted in Table 6. In Table 7, in turn, we show the resistances to every individual agent, as well as the individual degree, closeness, betweenness and eigenvalue scores. Here, all centrality computations have been undertaken systematically with UCINET (Borgatti et al., 2002).

Let us comment briefly the results. The most repeated components of the hard core are Marwan Al-Shehhi, Nawaf Al-Hazmi, Mohamed Atta, Waleed Al-Shehri and Hamza Al-Ghamdi, and hence all the cells are represented in this group. When running the algorithm, the first three mentioned hijackers appeared frequently in the first or second iteration, which is not surprising as Atta an al-Shehhi were reported to be leaders of the network, and Al-Hazmi had contact with members of all the cells. Al-Shehri and Al-Ghamdi represent the unique tie of some individuals to the rest of the network. Consequently, their presence in the hard core is necessary in order to allow the information to diffuse to these individuals; in fact, these are the only two nodes whose absence would disconnect the graph.

Observe that the resistances to Atta and Al-Shehhi are always very low and similar. Except in one case, the resistance to Al-Shehhi is a little bit smaller than that to Atta in the cases where the influence matrix depends exclusively on the distances of the graph, and this fact suggests a certain structural advantage of the position of the former. Notice that this advantage cannot be concluded from its closeness nor its betweeness, and in fact Atta becomes the main character when in $\mathbf{S}(10)$ we introduce the betweenness in the model. This happens again when we consider the exogenous information through models $\mathbf{S}(6)$ and $\mathbf{S}(7)$. It is also remarkable that, except in one instance, Atta appearing in the hard core causes Al-Shehhi to not appear, and viceversa. From this point of view the two terrorists share a great part of the influence.

Let us also comment some differences between the considered scenarios. We have already described how the weights drastically change the "leader" of the hard core. For $\mathbf{S}(1)$ to $\mathbf{S}(5)$, the weight matrices depend on the distance between nodes. In $\mathbf{S}(1)$ and $\mathbf{S}(3)$ only very short distances count, such that the hard core "requires" the presence of Waleed Al-Shehri and Hamza Al-Ghamdi in order to reach the outskirts. On the other hand, in $\mathbf{S}(2)$ and $\mathbf{S}(4)$, one of these is replaced by a more central node, namely Jarrah and Atta, as the time needed to arrive to the more distant nodes is smaller. Another very different point of view is given by $\mathbf{S}(5)$, where at least three of the hijackers score very high in degree and closeness in the selected 


\begin{tabular}{|c|c|c|c|c|c|c|c|c|c|c|c|c|c|c|}
\hline & $\mathbf{S}(1)$ & $\mathbf{S}(2)$ & $\mathbf{S}(3)$ & $\mathbf{S}(4)$ & $\mathbf{S}(5)$ & $\mathbf{S}(6)$ & $\mathbf{S}(7)$ & $\mathbf{S}(8)$ & $\mathbf{S}(9)$ & $\mathbf{S}(10)$ & Eig. & Deg. & Clos. & Betw. \\
\hline Mohamed Atta & 43.568 & 30.163 & 28.745 & 27.935 & 31.843 & 53.693 & 32.731 & 48.330 & 32.309 & 14.061 & 0.361 & 0.263 & 0.068 & 0.119 \\
\hline Marwan Al-Shehhi & 41.009 & 30.093 & 28.950 & 28.482 & 26.660 & 56.269 & 35.446 & 54.316 & 32.345 & 18.380 & 0.348 & 0.316 & 0.064 & 0.116 \\
\hline Said Jarrah & 47.592 & 31.383 & 31.819 & 30.569 & 28.883 & 66.288 & 55.408 & 58.509 & 36.626 & 44.471 & 0.334 & 0.263 & 0.061 & 0.116 \\
\hline Khalid Al-Midhar & 84.143 & 37.228 & 49.715 & 37.319 & 34.464 & 188.968 & 64.595 & 148.686 & 72.299 & $\infty$ & 0.173 & 0.105 & 0.051 & 0 \\
\hline Nawaf al-Hazmi & 42.292 & 28.523 & 26.817 & 27.299 & 27.648 & 65.532 & 47.846 & 26.106 & 30.632 & 20.977 & 0.405 & 0.368 & 0.068 & 0.170 \\
\hline Abdulaziz Al-Omari & 67.935 & 34.861 & 37.723 & 33.694 & 34.639 & 73.380 & 55.680 & 131.587 & 60.879 & 20.446 & 0.166 & 0.158 & 0.057 & 0.163 \\
\hline Waleed Al-Shehri & 145.999 & 40.733 & 52.911 & 53.511 & 41.146 & 148.241 & 50.755 & 465.507 & 171.035 & 43.720 & 0.042 & 0.158 & 0.043 & 0.116 \\
\hline Wail Al-Shehri & 201.605 & 49.194 & 67.583 & 88.248 & 49.823 & 184.932 & 52.427 & 822.615 & 301.641 & $\infty$ & 0.012 & 0.105 & 0.033 & 0 \\
\hline Majed Moqed & 140.545 & 42.642 & 54.129 & 46.168 & 42.103 & 167.989 & 69.717 & 558.813 & 139.393 & $\infty$ & 0.083 & 0.053 & 0.044 & 0 \\
\hline Salem Al-Hazmi & 62.853 & 33.936 & 34.749 & 33.134 & 32.655 & 109.774 & 57.885 & 87.155 & 49.717 & 194.906 & 0.241 & 0.158 & 0.058 & 0.008 \\
\hline Hami Hanjour & 47.487 & 29.909 & 27.296 & 29.115 & 29.955 & 83.160 & 40.527 & 45.770 & 34.818 & 21.272 & 0.377 & 0.316 & 0.064 & 0.093 \\
\hline Ahmed Al-Haznawi & 69.093 & 35.102 & 36.438 & 35.167 & 33.291 & 123.460 & 101.784 & 96.478 & 61.788 & 208.518 & 0.181 & 0.158 & 0.053 & 0.016 \\
\hline Satam Suqami & 201.537 & 49.227 & 67.570 & 88.199 & 49.821 & 206.315 & 78.924 & 824.928 & 302.793 & $\infty$ & 0.012 & 0.105 & 0.033 & 0 \\
\hline Said Al-Ghamdi & 64.864 & 33.702 & 33.625 & 33.788 & 37.118 & 143.069 & 78.350 & 41.136 & 55.330 & 300.037 & 0.230 & 0.211 & 0.054 & 0.006 \\
\hline Ahmed Al-Hami & 72.898 & 35.660 & 37.492 & 35.795 & 35.027 & 155.917 & 90.238 & 29.623 & 64.174 & $\infty$ & 0.197 & 0.158 & 0.052 & 0 \\
\hline Fayed Ahmed & 94.547 & 37.803 & 42.973 & 37.081 & 40.009 & 232.706 & 110.031 & 249.070 & 89.764 & 137.727 & 0.094 & 0.105 & 0.052 & 0.029 \\
\hline Mohand Al-Shahri & 98.647 & 38.891 & 44.349 & 40.353 & 40.027 & 194.741 & 113.224 & 235.037 & 100.057 & 216.080 & 0.077 & 0.105 & 0.047 & 0.019 \\
\hline Hamza Al-Ghamdi & 57.261 & 30.866 & 28.857 & 30.961 & 33.570 & 121.461 & 71.769 & 35.593 & 48.432 & 43.060 & 0.253 & 0.316 & 0.058 & 0.104 \\
\hline Ahmed Al-Ghamdi & 148.728 & 43.749 & 55.943 & 48.911 & 46.906 & 327.979 & 131.802 & 543.072 & 169.172 & $\infty$ & 0.056 & 0.053 & 0.041 & 0 \\
\hline
\end{tabular}

Table 7: Individual resistances and centralities

hard core. This is not surprising, as this weight matrix counts the number of geodesic paths. It neither comes as a surprise that the values of betweenness are lower: if there are a lot of edges "around" the hard core, there will always be alternative paths if a node is erased.

It is also interesting to observe that Al-Omari appears in the hard core only for two of the measures that integrate in the model the individual centralities of the hijackers. In other words, the relevance of this node is only perceived by these particular versions of the weight matrix, as it happens with Atta for $\mathbf{S}(6), \mathbf{S}(7)$ and $\mathbf{S}(10)$. From this facts it can be concluded that the flexibility of the model allows to identify leadership which does not follow straight from the graph structure.

This present approach allows to explore different features of the network in an unified way. Conversely, next to the differences, one may also wonder about the similarities of the results obtained according to the different models. After all, they are constructed following the same basic scheme. In order to address this question we have computed the correlations between the resistances (Table 8). Looking at them as individual measures of power, all of them that depend on the distance -including closeness- are highly correlated, and the values of the correlations descend drastically when we deal with $\mathbf{S}(6), \mathbf{S}(7)$ and $\mathbf{S}(10)$. This is consistent with the fact that we are introducing exogenous information, in the first two cases, and the independence of betweenness and distance, in the latter.

For obvious reasons one has to be careful with these correlations. Firstly, the small sample size affects their reliability, and secondly, the correlations do not take account for the fact that we are interested in relative power instead of the actual values of the measure. These orders show certain variations for the different measures (Table 9), which imply in turn significative changes in the outcomes of the greedy algorithm. Nevertheless, taking account of these 


\begin{tabular}{|c|c|c|c|c|c|c|c|c|c|c|c|c|c|}
\hline & $\mathbf{S}(1)$ & $\mathbf{S}(2)$ & $\mathbf{S}(3)$ & $\mathbf{S}(4)$ & $\mathbf{S}(5)$ & $\mathbf{S}(6)$ & $\mathbf{S}(7)$ & $\mathbf{S}(8)$ & $\mathbf{S}(9)$ & $\mathbf{S}(10)$ & Eig. & Deg. & Clos. \\
\hline $\mathbf{S}(1)$ & & & & & & & & & & & & & \\
\hline $\mathbf{S}(2)$ & 0.981 & & & & & & & & & & & & \\
\hline $\mathbf{S}(3)$ & 0.975 & 0.987 & & & & & & & & & & & \\
\hline $\mathbf{S}(4)$ & 0.946 & 0.916 & 0.908 & & & & & & & & & & \\
\hline $\mathbf{S}(5)$ & 0.954 & 0.961 & 0.929 & 0.871 & & & & & & & & & \\
\hline $\mathbf{S}(6)$ & 0.693 & 0.747 & 0.727 & 0.516 & 0.801 & & & & & & & & \\
\hline $\mathbf{S}(7)$ & 0.302 & 0.403 & 0.331 & 0.134 & 0.481 & 0.799 & & & & & & & \\
\hline $\mathbf{S}(8)$ & 0.983 & 0.952 & 0.950 & 0.937 & 0.916 & 0.624 & 0.215 & & & & & & \\
\hline $\mathbf{S}(9)$ & 0.981 & 0.950 & 0.942 & 0.988 & 0.919 & 0.602 & 0.211 & 0.973 & & & & & \\
\hline $\mathbf{S}(10)$ & 0.679 & 0.718 & 0.743 & 0.624 & 0.640 & 0.618 & 0.270 & 0.651 & 0.641 & & & & \\
\hline Eig. & -0.882 & -0.927 & -0.903 & -0.756 & -0.919 & -0.790 & -0.563 & -0.815 & -0.813 & -0.554 & & & \\
\hline Deg. & -0.716 & -0.822 & -0.803 & -0.549 & -0.786 & -0.786 & -0.614 & -0.658 & -0.610 & -0.623 & 0.888 & & \\
\hline Clos. & -0.954 & -0.978 & -0.960 & -0.878 & -0.949 & -0.776 & -0.479 & -0.896 & -0.913 & -0.694 & 0.952 & 0.820 & \\
\hline Bet. & -0.478 & -0.587 & -0.546 & -0.420 & -0.550 & -0.665 & -0.587 & -0.409 & -0.422 & -0.603 & 0.550 & 0.708 & 0.629 \\
\hline
\end{tabular}

Table 8: Correlations between individual resistances and centralities

subtleties, we observe in Table 6 similarities of hard cores. The explanation is that measures $\mathbf{S}(1)-\mathbf{S}(4)$ are based on distance, $\mathbf{S}(6)$ and $\mathbf{S}(7)$ rest upon the same weights, and the last three are constructed in a similar way taking account of the individual centralities. Same features of the network are likely to produce similarities, although, as illustrated by $\mathbf{S}(1)$ and $\mathbf{S}(2)$, this is not always the case.

As discussed in the previous section, however, the main focus of resistance is not individual power, but diffusion time and group influence. There we showed that the relation between the resistance of the society to groups and group centrality measures is not closed. The fact that the models produce different hard cores therefore does not come as a surprise.

It is also interesting to compare our results with those obtained from different versions of the key player algorithm of Borgatti (2006). Similar to our approach, this algorithm aims at identifying an influential group, but unlike the resistance it focuses on the maximally connected set of nodes (KPP-positive) or on the group of nodes whose deletion would cause a major disruption (KPP-negative). The results of the application of the algorithm to some relevant examples of the earlier defined influence matrices are depicted in table 10. There is a significant similarity in the selected members of the core (3 out of 4), although for the two different versions of the algorithm the members are not the same. In any case, this is remarkable because the key player power is usually highly related with betweenness, while the individual resistance is in all versions correlated to closeness (see Table 8 ) but not to betweenness: in particular, the correlation between resistance and betweenness is -0.478 , a non-meaningful value. Theoretically, small values of betweenness may be compatible with small values of the resistance of the society. This is the case if for some node there is a small distance to any other node in the network, but every path that passes through the node can be avoided using another. This suggests that the above coincidences may not be general, but merely a consequence of the small diameter of the network combined with high connectivity. 


\begin{tabular}{|c|c|c|c|c|c|c|c|c|c|c|c|c|c|c|}
\hline & $\mathbf{S}(1)$ & $\mathbf{S}(2)$ & $\mathbf{S}(3)$ & $\mathbf{S}(4)$ & $\mathbf{S}(5)$ & $\mathbf{S}(6)$ & $\mathbf{S}(7)$ & $\mathbf{S}(8)$ & $\mathbf{S}(9)$ & $\mathbf{S}(10)$ & Eig. & Deg. & Clos. & Betw. \\
\hline Mohamed Atta & 3 & 4 & 3 & 2 & 5 & 1 & 1 & 6 & 2 & 1 & 17 & 15 & 18 & 17 \\
\hline Marwan Al-Shehhi & 1 & 3 & 5 & 3 & 1 & 2 & 2 & 7 & 3 & 2 & 16 & 17 & 16 & 16 \\
\hline Said Jarrah & 5 & 6 & 6 & 5 & 3 & 4 & 7 & 8 & 5 & 8 & 15 & 14 & 15 & 12 \\
\hline Khalid Al-Midhar & 12 & 12 & 14 & 13 & 9 & 15 & 10 & 12 & 12 & 15 & 9 & 7 & 7 & 1 \\
\hline Nawaf al-Hazmi & 2 & 1 & 1 & 1 & 2 & 3 & 4 & 1 & 1 & 4 & 19 & 19 & 19 & 19 \\
\hline Abdulaziz Al-Omari & 9 & 9 & 11 & 8 & 10 & 5 & 8 & 11 & 9 & 3 & 8 & 9 & 12 & 18 \\
\hline Waleed Al-Shehri & 16 & 15 & 15 & 17 & 15 & 11 & 5 & 15 & 17 & 7 & 3 & 8 & 4 & 15 \\
\hline Wail Al-Shehri & 19 & 18 & 19 & 19 & 19 & 14 & 6 & 18 & 18 & 18 & 2 & 4 & 2 & 2 \\
\hline Majed Moqed & 15 & 16 & 16 & 15 & 16 & 13 & 11 & 17 & 15 & 16 & 6 & 2 & 5 & 3 \\
\hline Salem Al-Hazmi & 7 & 8 & 8 & 7 & 6 & 7 & 9 & 9 & 7 & 10 & 13 & 12 & 13 & 8 \\
\hline Hami Hanjour & 4 & 2 & 2 & 4 & 4 & 6 & 3 & 5 & 4 & 5 & 18 & 18 & 17 & 13 \\
\hline Ahmed Al-Haznawi & 10 & 10 & 9 & 10 & 7 & 9 & 16 & 10 & 10 & 11 & 10 & 10 & 10 & 9 \\
\hline Satam Suqami & 18 & 19 & 18 & 18 & 18 & 17 & 14 & 19 & 19 & 19 & 1 & 3 & 1 & 4 \\
\hline Said Al-Ghamdi & 8 & 7 & 7 & 9 & 12 & 10 & 13 & 4 & 8 & 13 & 12 & 13 & 11 & 7 \\
\hline Ahmed Al-Hami & 11 & 11 & 10 & 11 & 11 & 12 & 15 & 2 & 11 & 14 & 11 & 11 & 9 & 5 \\
\hline Fayed Ahmed & 13 & 13 & 12 & 12 & 13 & 18 & 17 & 14 & 13 & 9 & 7 & 6 & 8 & 11 \\
\hline Mohand Al-Shahri & 14 & 14 & 13 & 14 & 16 & 18 & 18 & 13 & 14 & 12 & 5 & 5 & 6 & 10 \\
\hline Hamza Al-Ghamdi & 6 & 5 & 4 & 6 & 8 & 8 & 12 & 3 & 6 & 6 & 14 & 16 & 14 & 14 \\
\hline Ahmed Al-Ghamdi & 17 & 17 & 17 & 16 & 17 & 19 & 19 & 16 & 16 & 17 & 4 & 1 & 3 & 6 \\
\hline
\end{tabular}

Table 9: Orderings according to individual measures

\begin{tabular}{|c|c|}
\hline KPP-Negative & KPP-Positive \\
\hline Al-Shehhi & Al-Shehhi \\
\hline Jarrah & Al-Shehri \\
\hline N. Al-Hazmi & Hanjour \\
\hline H. Al-Ghamdi & H. Al-Ghamdi \\
\hline
\end{tabular}

Table 10: Results of the key player algorithm

Remark 2. Note that in Table 9 the ordering of the last four columns is approximately reversed to the ordering of the rest, as power is conceptually inverse to resistance, and then the correlations are usually negative. Moreover, in Table 7 there are some resistances for $\mathbf{S}(10)$ (respectively betweenness) that are infinite (resp. zero), so in Table 9 the ordinal numbers 14-19 (resp. 1-6) are interchangeable.

\section{Conclusions}

This paper proposed a measure for a group's ability to trigger collective action and discussed the differences to other (centrality) measures. The Wolfe Primate Data and the data of the 11S hijackers' network illustrated how this measure comes to more detailed and different conclusions than those discussed in the literature on Graph Theory. The setup of our model is flexible to modifications when required by context and data. For example, in the context of revolutions the interest is typically the expected time it takes until the majority of agents takes action. Also in other contexts, as innovations, one might wonder about the most influential group in order 
to overturn a certain percentage $q$ of the society. Next, it would be interesting to improve the greedy heuristic used to find a powerful group, by means of an in-and-out procedure (as in Borgatti's key-player algorithm), a careful selection of the initial group, or taking account of the concept of the efficiency (see Everett and Borgatti, 1999). In this sense, it would also be desirable to find conditions which assure resistance's supermodularity, i.e., decreasing marginal effects, which are very important for a social planner who uses this analysis with respect for example disease prevention. Moreover, a controlled random selection of the initial nodes could allow to define a centralization measure. Finally, we intend to explore the consequences of integrating closely related nodes in a certain cluster, and relating the outcomes before and after this operation. In some sense, the latter could also be interpreted in the framework of network exposure as an exotic, but useful, way of defining the weight matrix.

\section{References}

[1] P. Allison (1984). Event history analysis, Newberry Park, Sage.

[2] C. Asavathiratham (2000). The influence model: a tractable representation for the dynamics of networked markov chains, Ph.D. Thesis, MIT.

[3] F. Bass (1969). A new product growth model for consumer durables. Management Science, 15, 215-227.

[4] R.L. Berger (1981). A necessary and sufficient condition for reaching a consensus using DeGroot's method. Journal of the American Statistical Association, 76, 415-418.

[5] P. Bonacich (1972). Factoring and weighting approaches to status scores and clique identification. Journal of Mathematical Sociology, 2, 113-120.

[6] P. Bonacich (1987). Power and centrality: a family of measures. American Journal of Sociology, 92, 1170-1182.

[7] S.P. Borgatti (2005). Centrality and network flow. Social Networks, 27, 55-71.

[8] S.P. Borgatti (2006). Identifying sets of key players in a social network. Computational and Mathematical Organization Theory, 12, 21-34.

[9] S.P. Borgatti, M.G. Everett and L.C. Freeman (2002). UCINET 6 for Windows: Software for Social Network Analysis. Harvard: Analytic Technologies.

[10] U. Brandes (2008). On variants of shortest-path betweenness centrality and their generic computation. Social Networks, 30, 136-145.

[11] S. Brin and L. Page (1998). The anatomy of a large-scale hypertextual Web search engine. Computer Networks and ISDN Systems, 30, 107-117. 
[12] J. Brown and P. Reinegen (1987). Social ties and word-of-mouth referral behavior. Journal of Consumer Research, 14, 950-362.

[13] R. Burt (1987). Social contagion and innovation: Cohesion versus structural equivalence. American Journal of Sociology, 92, 1287-1335.

[14] S. Chatterjee and E. Seneta (1977). Towards consensus: some convergence theorems on repeated averaging. Journal of Applied Probability, 14, 89-97.

[15] P. Clifford and A. Sudbury (1973). A model for spatial conflict. Biometrika, 60, 581-588.

[16] J.S. Coleman (1971). Control of Collectivities and the Power of a Collectivity to Act. In B. Lieberman (Ed.), Social Choice. Gordon and Breach, London, 269-300.

[17] J.S. Coleman (1990). Foundations of Social Theory, Harvard University Press.

[18] J. Conlisk (1976). Interactive Markov chains. Journal of Mathematical Sociology, 4, 157-185.

[19] M.H. DeGroot (1974) . Reaching a consensus. Journal of the American Statistical Association, 69, 118-121.

[20] P.S. Dodds and D.J. Watts (2005). A generalized model of social and biological contagion. Journal of Theoretical Biology, 232, 587-604.

[21] P. Doreian (1982). Maximum likelihood methods for linear models. Sociological Methods and Research, 10, 243-269.

[22] L. Erbring and A. Young (1979). Individuals and social structure: contextual effects as endogenous feedback. Sociological Methods and Research, 7, 396-430.

[23] E. Even-Dar and A. Shapira (2007). A note on maximizing the spread of influence in Social Networks. In X. Deng and F.C. Grabam (Eds.), WINE 2007, LNCS 4858. Springer-Verlag, Berlin Heidelberg, 281-286.

[24] M.G. Everett and S.P. Borgatti (1999). The centrality of groups and classes. Journal of Mathematical Sociology, 23, 181-201.

[25] L.C. Freeman (1979). Centrality in social networks: conceptual clarification. Social Networks, 1, 215-239.

[26] J.R.P. French, Jr. (1956). A formal theory of social power. The Psychological Review, 63, 181194.

[27] N. Friedkin (1991). Theoretical foundations for centrality measures. American Journal of Sociology, 96, 1478-1504. 
[28] N. Friedkin (1993). Structural bases of interpersonal influence in groups: a longitudinal case study. American Sociological Review, 58, 861-872.

[29] N. Friedkin (1998). A structural theory of social influence, Cambridge University Press.

[30] N. Friedkin (1999). Choice shift and group polarization. American Sociological Review, 64, 856-875.

[31] N. Friedkin (2001). Norm formation in social influence networks, Social Networks, 23, 167189.

[32] N. Friedkin (2010). The Attitude-Behavior Linkage in Behavioral Cascades. Social Psychology Quarterly, 73, 196-213.

[33] N. Friedkin and E. Johnsen (1990). Social influence and opinions. Journal of Mathematical Sociology, 15, 193-205.

[34] N. Friedkin and E. Johnsen (1997). Social positions in influence networks. Social Networks, 19, 210-222.

[35] N. Friedkin and E. Johnsen (1999). Social influence networks and opinion change. Advances in Group Processes, 16, 1-29.

[36] J. Goldenberg, B. Libai and E. Muller (2001). Talk of the network: a complex systems look at the underlying process of word-of-mouth. Marketing Letters, 12, 211-223.

[37] M. Granovetter (1978). Threshold models of collective behavior. American Journal of Sociology, 83, 1420-1443.

[38] F. Harary (1959). A criterion for unanimity in French's theory of social power. In: D. Cartwright (Ed.), Studies in Social Power. Institute for Social Research, Ann Arbor, MI, 168-182.

[39] R.A. Holley and T.M. Liggett (1975). Ergodic theorems for weakly interacting infinte systems and the voter model. Annals of Probability, 3, 643-663.

[40] L. Katz (1953). A new status index derived from sociometric analysis. Psychometrica, 18, $39-43$.

[41] D. Kempe, J. Kleinberg and E. Tardos (2005). Influential nodes in a diffusion model for social networks. In Proc. 32nd International Colloquium on Automata, Languages and Programming, 1127-1138.

[42] V. Krebs (2002). Mapping Networks of Terrorist Cells. Connections, 24, 43-52.

[43] D. Lopez-Pintado and D.J. Watts (2008). Social influence, binary decisions, and collective dynamics. Rationality and Society, 20(4), 399-443. 
[44] V. Mahajan, E. Muller and F. Bass (1990). New product diffusion models in marketing: a review and directions for research. Journal of Marketing, 54, 1-26.

[45] P. Marsden and N. Friedkin (1993). Network studies of social influence. Sociological Methods and Research, 22, 127-151.

[46] M.E.J. Newman (2001). Scientific collaboration networks. II. Shortest paths, weighted networks, and centrality. Physical Review E, 64, 016132.

[47] Y. Ni, L. Xie and Z-Q. Liu (2010). Minimizing the expected complete influence time of a social network. Information Sciences, 180, 2514-2527.

[48] T. Opsahl, F. Agneessensb and J. Skvoretzc (2010). Node centrality in weighted networks: Generalizing degree and shortest paths. Social Networks, 32, 245-251.

[49] M. Richardson and P. Domingos (2002). Mining knowledge-sharing sites for viral marketing. In Eighth International Conference on Knowledge Discovery and Data Mining.

[50] E.M. Rogers (1995). Diffusion of Innovations, fourth edition. New York: Simon and Schuster.

[51] T.C. Schelling (1971) Dynamic models of seggregation. Journal of Mathematical Sociology, 1, 143-186.

[52] W. Stadje (1997). Asymptotic bahavior of some interactive population flow models. The Annals of Applied Probability, 7, 837-854.

[53] T.W. Valente (1995). Network models of the diffusion of innovations. Cresskill, NJ: Hampton Press.

[54] T.W. Valente (1996). Social network thresholds in the diffusion of innovations. Social Networks, 18, 69-89.

[55] T.W. Valente (2005). Network models and methods for studying the diffusion of innovations. In P.J. Carrington and J. Scott and S. Wasserman (Eds.), Models and Methods in Social network Analysis. Cambridge University Press, Cambridge, 98-116.

[56] T.W. Valente (2010). Social Networks and Health. Models, methods, and applications. Oxford University Press.

[57] D. Watts (2002). A simple model of global cascades in random networks. In PNAS: Proc. Natnl. Acad. Sci., 99, 5766-5771.

[58] H.P. Young (2003). The diffusion of innovations in social networks. In Lawrence E. Blume and Steven D. Durlauf (Eds.), The Economy as a Complex Evolving System, vol. III. Oxford University Press. 